\title{
Magnetic properties of colloidal suspensions of interacting magnetic particles
}

\author{
B. Huke and M. Lücke \\ Institut für Theoretische Physik, \\ Universität des Saarlandes, \\ D-66041 Saarbrücken, Germany
}

(Dated: October 22, 2018)

\begin{abstract}
We review equilibrium thermodynamic properties of systems of magnetic particles like ferrofluids in which dipolar interactions play an important role. The review is focussed on two subjects: $(i)$ the magnetization with the initial magnetic susceptibility as a special case and (ii) the phase transition behavior. Here the condensation ("gas/liquid") transition in the subsystem of the suspended particles is treated as well as the isotropic/ferromagnetic transition to a state with spontaneously generated long-range magnetic order.
\end{abstract}

PACS numbers: 


\section{Contents}

I. Introduction

II. Statistical mechanical description

$\begin{array}{ll}\text { A. Ideal paramagnetism } & 7\end{array}$

B. Weiss and Onsager model]

$\begin{array}{ll}\text { C. Short-range potentials } & 9\end{array}$

III. Initial susceptibility

A. Cluster expansion methods 12

B. Hvpernetted chain and related approaches 15

$\begin{array}{ll}\text { C. Numerical simulations } & 18\end{array}$

D. A comparison 19

$\begin{array}{ll}\text { E. Other potentials } & 20\end{array}$

$\begin{array}{ll}\text { F. Polvdisperse theories } & 20\end{array}$

G. Applications to ferrofluids 21

$\begin{array}{ll}\text { IV. Magnetization } & 24\end{array}$

V. Nonspherical potentials and higher-order magnetic moments 30

VI. Phase transitions

A. Dilute/dense phase transitions

$\begin{array}{ll}\text { B. Ferromagnetic phases } & 37\end{array}$

VII. Conclusion $\quad 42$

$\begin{array}{ll}\text { Acknowledgments } & 44\end{array}$

$\begin{array}{ll}\text { References } & 45\end{array}$ 


\section{INTRODUCTION}

Ferrofluids [1] are suspensions of ferromagnetic or ferrimagnetic particles with diameters of the order of $10 \mathrm{~nm}$ in a liquid carrier. A common combination is magnetite $\left(\mathrm{Fe}_{3} \mathrm{O}_{4}\right)$ in hydrocarbons. The volume fraction of the magnetic material is typically a few percent. The particles are monodomain. They are too small for the build-up of domain walls [2, 3]. Thus, the quantum-mechanical exchange interaction between the electronic spins can keep all the atomic magnetic moments aligned so that the total magnetic moment, $m$, of the particles is of the order of about $10^{4} \mu_{B}$. Hence, to describe the equilibrium magnetic properties of the ferrofluid one can take each particle $i$ to have a magnetic moment $\mathbf{m}_{i}(t)$ that fluctuates in time, however, with its magnitude $m_{i}=\left|\mathbf{m}_{i}(t)\right|$ being fixed. Thus, we do not address here the magnetic properties of the ferrofluid system on the nm scale, i.e., of the magnetic particles themselves: Questions related to the size and form of the magnetocrystalline anisotropy, to magnetostrictive effects, to shape anisotropy, to dead layers etc. of the particles [2, 3, 4, 15] are not addressed here.

Whether the magnetic moment $\mathbf{m}_{i}(t)$ fluctuates only as a result of the rotational diffusion of the particle in the carrier liquid (Brownian relaxation) or also because the temperature is sufficiently high to stimulate $\mathbf{m}_{i}(t)$ itself to move in the potential landscape over the anisotropy energy barriers of the particle's lattice structure (Néel relaxation) does not influence the long-time equilibrium magnetic properties of the suspension but only its dynamics. In any case the magnetic particles cause the ferrofluid to display the equilibrium thermodynamics of a paramagnetic liquid - if one postpones for the moment the currently debated question of whether spontaneous long-range magnetic order is possible or not. The initial susceptibility can easily reach values of $\chi \approx 1$ and higher. Being orders of magnitude larger than for ordinary (molecular) paramagnetic liquids one thus speaks of a "superparamagnetic" liquid.

Ferrofluid particles are small enough to avoid segregation caused by gravity or customary magnetic gradient fields. But the strong van der Waals attraction between the nanoscale particles would lead to irreversible agglomeration. To prevent this, they are either coated with polymer surfactants of about $2 \mathrm{~nm}$ length whose entropic repulsion counteracts the van der Waals forces, or with ionic groups that prevent agglomeration due to their electrostatic repulsion. In the latter case the carrier is a polar liquid like water, containing the necessary 
counterions.

In a sufficiently diluted ferrofluid the magnetic particles can be thought as noninteracting, and the equilibrium magnetic properties of such a ferrofluid are those of an ideal paramagnetic gas. Still, there is an important difference to a molecular paramagnetic fluid like, say, oxygen: the ferrofluid particles are not identical, they differ both in size and magnetic moment. This polydispersity has influence on the properties of the ferrofluid, in particular also on the equilibrium magnetization.

At higher concentrations the ideal gas approximation fails to reproduce the equilibrium magnetization curve of ferrofluids. This is already the case for susceptibilities of the order of unity. Then, the particle interaction — and first of all the dipolar interaction — has to be taken into account. Since there exists a perfect analogy between magnetic and electric dipoles, the large number of theories developed for the latter case are directly applicable to this problem.

The thermodynamics of dipolar interacting particles is not only of interest because of the applicability to (electric) polar fluids and ferrofluids. It is also of large theoretical interest because the dipole-dipole interaction shows at least two interesting features: $(i)$ it is attractive and/or repulsive, depending on the orientation of the particles and ( $i i)$ it is of long-range nature.

Because the dipolar interaction is neither always attractive nor always repulsive it is not trivially clear how the magnetic moments of the particles affect, e. g., the condensation phase transition of the suspended particles and especially whether the dipole-dipole interaction can cause such a transition in a system of particles without additional attractive interactions. This question is still being discussed in the literature. An externally applied magnetic field can be expected to further modify the phase transition behavior.

Another example of a phase transition where the magnetic moments play an important role is the isotropic/ferromagnetic transition, i.e., a spontaneous breaking of the rotational symmetry resulting in a nonvanishing magnetization without applied magnetic field. Also this question whether the dipolar interaction alone can trigger such a phase transition is not yet settled.

There is quite an extensive literature on ferrofluids. The references to it are accumulated in the special issues of the Journal of Magnetism and Magnetic Materials that cover the International Conferences on Magnetic Fluids. Odenbach et al. [6] show in the latest 
issue that by 2002 the total number of papers on ferrofluids has grown to well above 6000 with about 1000 being published in the period 1998-2001 7]. The research topics cover problems as different as fluid dynamics and the modifications in classical pattern formation experiments by using ferrofluids, pattern formation processes genuine to ferrofluids as the Rosensweig instability or the labyrinth instability, magnetorheological properties, dynamical magnetic susceptibility, medical and engineering applications [8, 9, 10, 11, 12].

We restrict ourselves here to the topics where the dipolar interaction between the magnetic particles is of major importance, namely the already mentioned equilibrium magnetization and initial susceptibility on the one hand, and phase transitions on the other hand.

The review is organized as follows. Following this introduction we discuss in Sec. [I] basic statistical mechanics of systems of dipolar particles. In Sec. III the influence of the dipolar interaction on the initial susceptibility of systems of otherwise spherical symmetric particles is reviewed, mainly for dipolar hard spheres and Lennard-Jones particles with an additional magnetic moment (Stockmayer particles). This question has already been discussed for the electrical case before ferrofluids became an active research area. The more general question of the equilibrium magnetization on the other hand was mainly investigated with regard to the application to ferrofluids. The literature concerning the equilibrium magnetization is reviewed in Sec. IV] In Sec. V] the effect of additional nonspherical interactions other than the dipolar one are briefly discussed. Phase transitions of the gas/liquid and isotropic/ferromagnetic type are reviewed in Sec. VI. We conclude in Sec. VII

Because of the analogy between systems of magnetically and electrically polar particles and for reasons of simplicity, we will always use the magnetical terminology when reviewing the literature, even when only the electric case is considered. This is mostly the case in the older literature. 


\section{STATISTICAL MECHANICAL DESCRIPTION}

A complete description of ferrofluids within the framework of statistical mechanics would have to include all the components of a ferrofluid: magnetic particles, carrier liquid, polymer surfactants etc. But theoretical approaches to explain the thermodynamic properties connected to magnetism generally concentrate quite successfully on the magnetic component. Since the magnetic properties of the carrier liquid can be neglected, it is clear that it can only have indirect influence on the magnetization. A more elaborate model of a ferrofluid describing both the magnetic particles and the carrier fluid is discussed by Kalikmanov [13], who shows that under some assumptions the carrier fluid has no influence on the equilibrium magnetic properties of the ferrofluid as a whole.

Ignoring the carrier, the ferrofluid can be described as a system of particles, $i=1 \ldots N$, each carrying a magnetic moment $\mathbf{m}_{i}$ and thus interacting with an external magnetic field $\mathbf{H}_{e}$ via the potential

$$
V_{i}=-\mathbf{m}_{i} \cdot \mathbf{H}_{e}
$$

The magnetic moments as used here include the vacuum permeability $\mu_{0}$, see e.g. Eq. (2.4). The absolute values $\left|\mathbf{m}_{i}\right|$ are fixed; but in general they are different from each other. These magnetic particles interact pairwise with each other via the dipole-dipole interaction potential

$$
V_{i j}^{D D}=-\frac{3\left(\mathbf{m}_{i} \cdot \hat{\mathbf{r}}_{i j}\right)\left(\mathbf{m}_{j} \cdot \hat{\mathbf{r}}_{i j}\right)-\mathbf{m}_{i} \cdot \mathbf{m}_{j}}{4 \pi \mu_{0} r_{i j}^{3}} .
$$

Here $\mathbf{r}_{i j}=r_{i j} \hat{\mathbf{r}}_{i j}$ is the distance vector between particles $i$ and $j$ (see Fig. 1). Since this potential decreases radially only as $r^{-3}$ the dipolar interaction is of long-range nature.

In addition to (2.2) there generally exist also some short-range interactions between the particles that we capture by the potential $V_{i j}^{S R}$. It might include nonmagnetic components and in general also short-range magnetic interactions involving quadrupolar or higher order magnetic moments. For later use we define the dimensionless potentials

$$
v_{i}=\frac{V_{i}}{k_{\mathrm{B}} T}, v_{i j}^{D D}=\frac{V_{i j}^{D D}}{k_{\mathrm{B}} T}, v_{i j}^{S R}=\frac{V_{i j}^{S R}}{k_{\mathrm{B}} T} .
$$

The long-range nature of the magnetic interaction requires some care in statistical mechanical considerations. The local magnetic field acting on a particle is the sum of the external field and the dipolar fields of the other particles. The dipolar contribution depends on the distribution of the particles on a macroscopic scale, i. e. in particular also on the 
probe geometry. In macroscopic magnetostatics this property translates into the fact that the equilibrium magnetization $\mathbf{M}$ of a magnetic medium depends on the internal field $\mathbf{H}$. This macroscopic field depends itself on $\mathbf{H}_{e}, \mathbf{M}$, and geometry. The difference between $\mathbf{H}$ and $\mathbf{H}_{e}$ can therefore be interpreted as an effect of dipolar interaction.

\section{A. Ideal paramagnetism}

If all dipolar interactions are discarded then the magnetic particles of the ferrofluid feel only the external magnetic field, i.e., $H=H_{e}$. This is an useful approximation if $M \ll H_{e}$ such that $H \approx H_{e}$. Assuming in addition all particles to be identical - the monodisperse case - with common magnetic moment $\left|\mathbf{m}_{i}\right|=m$, then the equilibrium magnetization is given by

$$
M=M_{L}=\frac{m N}{\mu_{0} V} \mathcal{L}\left(\frac{m H}{k_{\mathrm{B}} T}\right)=M_{\text {sat }} \mathcal{L}(\alpha)
$$

with $H=H_{e}$. Here $N / V$ is the particle density and $\mathcal{L}$ is the Langevin function, $\mathcal{L}(\alpha)=$ $\operatorname{coth}(\alpha)-1 / \alpha$. Its argument $\alpha=m H / k_{\mathrm{B}} T$ measures the energy of the moment $m$ in the field $H=H_{e}$ in units of the thermal energy $k_{\mathrm{B}} T$. The saturation magnetization $M_{\text {sat }}=m N / \mu_{0} V$ of the ferrofluid corresponds to the case of parallel dipoles.

One might think that using in Eq. (2.4) the real internal field $H$ in the ferrofluid instead of $H_{e}$ would be an improvement of the ideal paramagnetic model by itself. However such a replacement does not incorporate the effect of dipolar interactions in a systematic and controlled manner since they additionally modify the simple functional relation (2.4) between equilibrium magnetization $M$ and internal field $H$ in various ways that are of the same order as the replacement of $H_{e}$ by $H$ in Eq. (2.4).

In a polydisperse extension of the above described ideal, noninteracting monodisperse model the magnetic moments of the particles are allowed to be different. Then the magnetization is given by

$$
M=M_{L}=\sum_{i} \frac{m_{i}}{\mu_{0} V} \mathcal{L}\left(\frac{m_{i} H}{k_{\mathrm{B}} T}\right) .
$$

Here the sum extends over all magnetic particles, $i=1, \ldots, N$. Using the mean magnetic moment $\bar{m}=(1 / N) \sum m_{i}$ to introduce the reduced moment $\mu_{i}=m_{i} / \bar{m}$ of particle $i$ we can write more conveniently

$$
M=\frac{\bar{m}}{\mu_{0} V} \sum_{i} \mu_{i} \mathcal{L}\left(\frac{\mu_{i} \bar{m} H}{k_{\mathrm{B}} T}\right)=M_{\text {sat }} \mathcal{L}^{\text {poly }}(\bar{\alpha})
$$


where

$$
M_{\text {sat }}=\frac{\bar{m} N}{\mu_{0} V}, \quad \mathcal{L}^{\text {poly }}(\bar{\alpha})=\frac{1}{N} \sum_{i} \mu_{i} \mathcal{L}\left(\mu_{i} \bar{\alpha}\right), \quad \bar{\alpha}=\frac{\bar{m} H}{k_{\mathrm{B}} T}
$$

\section{B. Weiss and Onsager model}

The earliest model of a selfinteracting magnetic medium is the mean-field Weiss model 14]. In this monodisperse model every particle is thought to be located in the center of an empty spherical cavity that is surrounded by a magnetic continuum with an internal field $H$ and magnetization $M$. In thermal equilibrium, the magnetization is given by a Langevin function into which enters the local field $H_{\text {local }}=H+M / 3$ within the spherical cavity. This leads to the implicit relation

$$
M=M_{\text {sat }} \mathcal{L}\left[\frac{m}{k_{\mathrm{B}} T}\left(H+\frac{M}{3}\right)\right],
$$

that can be solved numerically to give the sought after equilibrium magnetization $M(H)$.

The initial susceptibility of the Weiss model

$$
\chi=\frac{\partial M}{\partial H}(H=0)=\frac{\chi_{L}}{1-\chi_{L} / 3},
$$

is equivalent to the well known Clausius-Mosotti relation. Here

$$
\chi_{L}=\frac{\partial M_{L}}{\partial H}(H=0)=\frac{1}{3} M_{s a t} \frac{m}{k_{\mathrm{B}} T}
$$

is the Langevin initial susceptibility of the ideal monodisperse paramagnetism (2.4).

The Weiss model works well for weakly interacting ferrofluids but strongly overestimates the magnetization of concentrated magnetic fluids. It predicts ferromagnetic solutions, i. e., a spontaneous magnetization $M(H=0) \neq 0$ whenever $\chi_{L}>3$. While this criterion is at odds with experiments the possible existence of ferromagnetic phases in systems of dipolar hard spheres cannot be ruled out for stronger interactions (c.f. Sec. VIB).

A similar early approach to the problem of a selfinteracting magnetic medium is the Onsager theory [15] originally conceived for polarizable molecules. Therein, say, spherical molecules occupy a cavity in polarizable continuum with susceptibility $\chi$. The field acting on the molecule is the sum of a cavity field plus a reaction field that is parallel to the actual total (permanent and induced) moment of the molecule. The resulting equation between $\chi_{L}$ and $\chi$ reads here

$$
\frac{\chi(3+2 \chi)}{3(1+\chi)}=\chi_{L}
$$


While the Weiss model overestimates the initial susceptibility of concentrated ferrofluids, the Onsager model generally underestimates it. It is instructive to write $\chi$ according to (2.9) and (2.11) for small $\chi_{L}$ as power series in $\chi_{L}$

$$
\chi=\chi_{L}+\frac{1}{3} \chi_{L}^{2} \pm \frac{1}{9} \chi_{L}^{3}+\ldots
$$

Here the positive sign refers to the Weiss model and the negative one to the Onsager model. The first and second term agree with a systematic expansion of $\chi$ in terms of dipolar coupling strength and magnetic volume fraction (c.f. Sec. IIIA). However, the third order term of this expansion reads $+\frac{1}{144} \chi_{L}^{3}$ (see Eq. [3.8). Thus both, Onsager as well as Weiss model strongly overestimate the influence of this term.

\section{Short-range potentials}

If one wants to go beyond the mean field models of Sec. IIB one has to specify the potential with which the magnetic particles interact besides the long-range dipolar one.

Rosensweig 1] has proposed a quite elaborate potential for the short-range interaction, $V^{S R}$, of sterically stabilized magnetic particles. It consists of the sum of the van der Waals attraction between spherical magnetic particles and the sterical repulsion of their surfactants, i.e., $V^{S R}=V^{\mathrm{vdW}}+V^{\text {ster }}$. The van der Waals attraction between the spheres is given by

$$
V_{i j}^{\mathrm{vdW}}\left(r_{i j}\right)=-\frac{A}{6}\left[\frac{2}{l^{2}+4 l}+\frac{2}{(l+2)^{2}}+\ln \frac{l^{2}+4 l}{(l+2)^{2}}\right] .
$$

Here $A$ is the Hamaker constant. The quantity

$$
l=\frac{2 s}{D_{\text {core }}}=\frac{2 r_{i j}}{D_{\text {core }}}-2
$$

is given by twice the surface-to-surface distance $s$ of the particles reduced by the diameter $D_{\text {core }}$ of the magnetic particles while $r_{i j}$ is the distance between their centers. The sterical repulsion of the surfactants is described by

$$
V_{i j}^{\text {ster }}\left(r_{i j}\right)=\frac{\pi D_{\text {core }}^{2} \xi k_{\mathrm{B}} T}{2}\left[2-\frac{l+2}{t} \ln \left(\frac{1+t}{1+l / 2}\right)-\frac{l}{t}\right] \quad 0<l<2 t .
$$

Here $\xi$ is the surface density of the polymers and

$$
t=\frac{2 \delta_{\text {polymer }}}{D_{\text {core }}}
$$


is given by twice the length, $\delta_{\text {polymer }}$, of the surfactant polymers. In the Rosensweig potential the carrier liquid component is not completely ignored but rather taken into account on a continuum level via the electric permeability of the liquid that enters into the Hamaker constant $A$.

Fig. 2 shows the interaction potential $V^{S R}=V^{\mathrm{vdW}}+V^{\text {ster }}$ (2.13, 2.15) for typical values given by Rosensweig: $A=10^{-19} \mathrm{~J}, \xi=1 \mathrm{~nm}^{-2}, D_{\text {core }}=10 \mathrm{~nm}$, and $\delta_{\text {polymer }}=2 \mathrm{~nm}$. The need for sterical stabilization becomes apparent in this plot. The van der Waals interaction is strongly attractive for small particle separations, it even diverges in this simple model. The sterical potential modifies the interaction for distances less than $4 \mathrm{~nm}$, such that only a small attractive tail at larger distances remains in the combined potential. At very small surface-to-surface separations the potential still diverges, but this inner attractive region is effectively shielded by a potential barrier with a height of about $25 k_{\mathrm{B}} T$.

To obtain an equivalent model potential for electrostatically stabilized ferrofluids, $V^{\text {ster }}$ is replaced (see e. g. [16]) by the interaction potential

$$
V_{i j}^{\text {Debye }}\left(r_{i j}\right)=\frac{Q_{\text {eff }}^{2}}{4 \pi \epsilon r_{i j}} e^{-\left(r_{i j}-D_{\text {core }}\right) / \lambda_{d}}
$$

of charged spheres in ionic solutions. Here $\lambda_{d}$ is the Debye length, $\epsilon$ is the dielectric constant of the carrier liquid, and $Q_{\text {eff }}$ is an effective charge of the particles. The combined potential shows qualitatively the same features as in the sterical case.

Another important model potential for describing dipolar particles is the Stockmayer potential in which the short-range interaction is given by the Lennard-Jones function for the van der Waals interaction of point-like particles

$$
v_{i j}^{S R}=4 v_{0}\left[\left(\frac{D}{r_{i j}}\right)^{12}-\left(\frac{D}{r_{i j}}\right)^{6}\right] .
$$

Here $D$ is the collision parameter marked by the zero of this potential and $v_{0}$ is the depth of the potential well.

However, most often the effects of particle interaction are discussed within the simpler model of dipolar hard spheres (DHS). Therein the short-range part of the interaction is given by the hard sphere repulsion potential

$$
v_{i j}^{S R}=v_{i j}^{H C}=\left\{\begin{array}{ll}
\infty & \text { for } r_{i j}<D \\
0 & \text { for } r_{i j}>D
\end{array},\right.
$$


with $D$ being the common hard sphere diameter in the monodisperse case. In the polydisperse case with different hard sphere diameters, $D_{i}$, one has to replace $D$ by $\left(D_{i}+D_{j}\right) / 2$ in the above equation. The only other interaction in this model is the long-range dipolar potential.

Some aspects of the sterical interaction (2.15) can also be incorporated into the DHS model. Since the magnetic field of a perfect, homogeneously magnetized sphere is exactly dipolar one can introduce into the DHS model particles that consist of two concentric spheres: an outer hard sphere with diameter $D$ and a magnetized spherical core of diameter $D_{\text {core }}<D$. The latter is the source of the dipole field. The nonmagnetic layer models the surfactants, i.e., $D-D_{\text {core }} \approx 2 \delta_{\text {polymer. }}$. A nonmagnetic dead layer [17] on the particle can also be modeled.

The DHS model system was initially introduced to describe the electric polarizability of fluids of polar molecules. However, in reality deviations from the spherical shape, particle polarizability, and higher order moments have an important influence. Ferrofluids, on the other hand, resemble much better the model system. That the hard sphere repulsion is a good approximation to the Rosensweig potential becomes apparent when looking at the quantity $\exp \left(-v^{S R}\right)$ which is more relevant for the equilibrium properties than $v^{S R}$ itself. In Fig. [3] $\exp \left[-v^{S R}(r)\right]$ is plotted for the Rosensweig potential (solid line) ignoring the irrelevant inner attractive region. As can be seen, $\exp \left(-v^{S R}\right)$ resembles quite well the unit step function $\exp \left(-v^{H C}\right)$ of the hard sphere potential that goes from 0 to 1 at contact distance.

The long dashed line for the best fitting hard sphere potential and the short dashed line for the van der Waals potential are included in Fig. [3 for comparison. Although the Rosensweig potential resembles more the van der Waals potential since both have an attractive part, the hard sphere potential fits the Rosensweig potential even a little bit better. 


\section{INITIAL SUSCEPTIBILITY}

Most of the older approaches to the magnetic or electric properties of polar fluids deal with the linear response problem, i. e. the initial susceptibility. The nonlinear behavior was of lesser interest in common atomic polar fluids since available electromagnetic fields were not strong enough to reach saturation. However, with the advent of superparamagnetic ferrofluids with large magnetic moments of the particles the linear and also the nonlinear magnetization behavior of interacting dipoles became again a more active research area.

Most of the approaches to calculate the initial susceptibility can roughly be classified into cluster expansion, hypernetted chain, and numerical simulation methods. In this section, we will mainly address the results for dipolar hard spheres.

\section{A. Cluster expansion methods}

Consider a system of $N$ particles interacting with each other via a pair potential $v_{i j}$ and with an external potential $v_{i}$. Then the canonical partition function is given by

$$
Z=\int \exp \left(-\sum_{i} v_{i}-\sum_{i<j} v_{i j}\right) \mathrm{d} \Gamma .
$$

Here $d \Gamma$ indicates the integration over the configuration space spanned by the positions and magnetic moments of the particles. The ideal-gas factor contained in the canonical partition function that comes from the momentum degrees of freedom is not indicated here.

The key point of the cluster expansion method is to introduce into (3.1) the functions

$$
f_{i j}=\exp \left(-v_{i j}\right)-1
$$

which are small when the interaction is weak. Thus, one inserts $\exp \left(-v_{i j}\right)=1+f_{i j}$ into (3.1) and expands the integrand in terms of $f_{i j}$.

Then (3.1) reads up to first order for example

$$
Z=Z_{N}^{0}+Z_{N-2}^{0} \sum_{i<j} \int f_{i j} \mathrm{~d}(i) \mathrm{d}(j),
$$

where $Z_{N}^{0}$ is the partition function of the ideal noninteracting $N$-particle system. These approximations to $Z$ then allow to calculate the thermodynamic quantities of interest. The advantage of this expansion is that in contrast to (3.1) the approximations to $Z$ such as 
(3.3) and higher orders require only low dimensional integrations, that can be performed at least numerically.

In the case of DHS the calculation of the leading terms can be done even analytically if one performs another series expansion, namely the expansion of (3.2) in terms of the dipolar interaction potential $v_{i j}^{D D}$ that enters via $v_{i j}=v_{i j}^{D D}+v_{i j}^{H C}$. The two expansions then translate into a power series for $Z$ and thus for $M$ or $\chi$ in two dimensionless parameters, namely the volume fraction

$$
\phi=\frac{N \pi D^{3}}{6 V}
$$

of the particles, and a dipolar coupling constant

$$
\lambda=\frac{m^{2}}{4 \pi \mu_{0} D^{3} k_{\mathrm{B}} T} .
$$

It is also common to choose $\rho^{*}=N D^{3} / V$ and $y=8 \phi \lambda / 3$ as parameters. These parameters fully characterize the system for $H=0$. To make a comparison with Stockmayer particles easier, we will use these parameters here also. In the case of Stockmayer particles one also has to specify the potential strength $v_{0}$ entering into Eq. (2.18). It should be noted that in the literature a system of Stockmayer particles is normally characterized by giving values for $1 / v_{0}$ and $\lambda / v_{0}$.

In ordinary ferrofluids, $\phi$ is of the order of $10^{-2}$. For magnetite ferrofluids with $10 \mathrm{~nm}$ particle diameters and an additional polymer layer of $2 \mathrm{~nm} \lambda$ is less than one. In this case, higher orders in the $(\phi, \lambda)$-expansion have only minor influence on $\chi$. But it is also possible to produce much stronger interacting ferrofluids. For example, van Ewijk, Vroege, and Philipse [18] report the production of ferrofluids with values for $\lambda$ up to 2.7 and volume fractions up to 0.5. Even higher $\lambda$ could be realized by Mamiya, Nakatani, and Furubayashi [19].

The long-range nature of the dipolar interaction causes some cluster integrals to be geometry dependent and mathematically ambiguous if the thermodynamic limit is naively performed. This problem is circumvented by dealing with a macroscopic but finite spherical geometry. In that case the initial susceptibility with respect to the external field $\chi_{e}=\partial M / \partial H_{e}\left(H_{e}=0\right)$ can be calculated unambiguously and is related to the geometryindependent quantity $\chi$ via

$$
\frac{\chi}{3+\chi}=\frac{1}{3} \chi_{e}
$$


In 1966 Jepsen [20] performs a cluster expansion to obtain

$$
\frac{\chi}{3+\chi}=\frac{1}{3} \chi_{L}-\frac{5}{144} \chi_{L}^{3}
$$

for DHS. $\chi$ is then up to the calculated order given by

$$
\chi=\chi_{L}+\frac{1}{3} \chi_{L}^{2}+\frac{1}{144} \chi_{L}^{3}
$$

This equation can also be understood as a $(\phi, \lambda)$-expansion, since $\chi_{L}=M_{\text {sat }} m / 3 k_{\mathrm{B}} T=$ $N m^{2} / 3 \mu_{0} k_{\mathrm{B}} T V$ can be written as

$$
\chi_{L}=8 \phi \lambda
$$

The fact that both the Weiss and the Onsager model correctly predict the prefactor of the $\chi_{L}^{2}$-term shows that this term has a mean-field origin being independent of the type of short-range interaction.

Rushbrooke [21] calculates more terms in 1979 . His result, as corrected later by Joslin 22], reads

$$
\begin{aligned}
& \frac{\chi}{3+\chi}= \frac{8}{3} \phi \lambda+\frac{64}{225} \phi^{2} \lambda^{4}-\frac{160}{9} \phi^{3} \lambda^{3}-\frac{8(1187-600 \ln 2)}{3375} \phi^{3} \lambda^{4}+1.13358 \phi^{4} \lambda^{3} \\
&= 2.66667 \phi \lambda+0.284444 \phi^{2} \lambda^{4}-17.7778 \phi^{3} \lambda^{3} \\
&-1.82782 \phi^{3} \lambda^{4}+1.13358 \phi^{4} \lambda^{3} .
\end{aligned}
$$

The $\phi \lambda$ - and $\phi^{3} \lambda^{3}$-terms agree with the $\chi_{L^{-}}$and $\chi_{L}^{3}$-terms of Jepsen. The last term requires a numerical integration. Rushbrookes error affected the $\phi^{3} \lambda^{4}$-term. Buckingham and Joslin [23] calculate the second dielectric virial coefficient, i. e. the $\phi^{2} \lambda^{n}$-terms in the above expansion:

$$
\frac{\chi}{3+\chi}=\ldots+\sum_{n=1}^{\infty} \frac{64}{3 n[(2 n+3) ! !]^{2}} \sum_{k=0}^{n}(3 k-n)\left(\begin{array}{c}
2 k \\
k
\end{array}\right) \phi^{2} \lambda^{2 n+2}+\ldots
$$

Joslin [22] presents besides the aforementioned correction to the result of Rushbrooke also the term

$$
\frac{32(315 \ln 2-218)}{3375} \phi^{3} \lambda^{5}=0.00323662 \phi^{3} \lambda^{5}
$$

Thereafter Rushbrooke and Shrubsall [24] calculate in addition the term

$$
\left[\frac{105133619}{4042500}-\frac{298976}{7875} \ln 2\right] \phi^{3} \lambda^{6}=-0.308396 \phi^{3} \lambda^{6} .
$$


A numerical calculation of the $\phi^{3}$-term as a function of $\lambda$ using Monte-Carlo methods is reported by Joslin and Goldman in 1993 [25].

Tani et al. 26] take a slightly different road to calculate $\chi$ using the system of (nonpolar) hard spheres as reference system. They get

$$
\chi=8 \phi \lambda+\frac{64}{3} \phi^{2} \lambda^{2}+f(\phi) \lambda^{2}
$$

where the last term is a numerical expression based on approximate expressions for the two- and three-particle correlation functions for hard spheres as found in Monte-Carlo simulations. For small $\phi$ this result reduces to (3.8). Goldman [27] compares results of this theory very successfully to Monte-Carlo data for systems of hard spheres and Stockmayer particles.

\section{B. Hypernetted chain and related approaches}

Using the hypernetted chain (HNC) approach the initial susceptibility is calculated via the two-particle correlation functions $g(1,2)$ and $h(1,2)=g(1,2)-1$ of the polar particles 28]. The HNC is based on the Ornstein-Zernike relation

$$
h(1,2)=c(1,2)+\frac{N}{4 \pi V} \int c(1,3) h(3,2) \mathrm{d}(3),
$$

defining the direct correlation function $c(1,2)$ and a closure relation, the HNC approximation reading

$$
\begin{array}{rc}
c(1,2)=h(1,2)-\ln [g(1,2)]-v_{12} & r_{12}>D, \\
g(1,2)=0 & r_{12}<D
\end{array}
$$

in the case of hard spheres. It can be shown [28] that this approximation is equivalent to a cluster expansion using an infinite number of $f$-integrals, neglecting only a certain class, the so-called bridge diagrams that appear only in higher orders.

Wertheim [29] and Nienhuis and Deutch [30] used the so-called mean spherical approximation (MSA) 31] to calculate the initial susceptibility of DHS. In the MSA (3.16) is replaced by

$$
c(1,2)=-v_{12} \quad \text { for } r_{12}>D
$$


effectively using the approximation $\ln [g(1,2)]=\ln [1+h(1,2)] \approx h(1,2)$, which is correct for large distances, where $h(1,2)$ is small. Wertheim showed that this equation together with (3.15) allows a simple ansatz, representing the dependence of the correlation functions on $\mathbf{m}_{1}$ and $\mathbf{m}_{2}$ as linear combinations of three functions. He sets

$$
h(1,2)=h_{S}\left(r_{12}\right)+h_{D}\left(r_{12}\right) f_{D}\left(\hat{\mathbf{m}}_{1}, \hat{\mathbf{m}}_{2}\right)+h_{\Delta}\left(r_{12}\right) f_{\Delta}\left(\hat{\mathbf{m}}_{1}, \hat{\mathbf{m}}_{2}\right)
$$

with

$$
\begin{aligned}
& f_{\Delta}\left(\hat{\mathbf{m}}_{1}, \hat{\mathbf{m}}_{2}\right)=\hat{\mathbf{m}}_{1} \cdot \hat{\mathbf{m}}_{2}, \\
& f_{D}\left(\hat{\mathbf{m}}_{1}, \hat{\mathbf{m}}_{2}\right)=3\left(\hat{\mathbf{r}}_{12} \cdot \hat{\mathbf{m}}_{1}\right)\left(\hat{\mathbf{r}}_{12} \cdot \hat{\mathbf{m}}_{2}\right)-\hat{\mathbf{m}}_{1} \cdot \hat{\mathbf{m}}_{2} .
\end{aligned}
$$

$g(1,2)$ and $c(1,2)$ have a similar representation with the same $f_{D}, f_{\Delta}$. The MSA then provides an implicit solution for $\chi$ that is given by the three equations

$$
\chi=\frac{\chi_{L}}{q(-x)}, \quad \chi_{L}=q(2 x)-q(-x), \quad q(x)=\frac{(1+2 x)^{2}}{(1-x)^{4}} .
$$

Here, $\chi$ depends only on $\chi_{L}=8 \phi \lambda$ but not on $\phi$ and $\lambda$ separately. Expanding $\chi$ in powers of $\chi_{L}$ yields again (3.8) for the leading terms.

Verlet and Weis 32] propose in 1974 an improved theory. Therein they replace the term $h_{S}(r)$ that does not depend on the dipolar character of the fluid by a better result for nonpolar hard spheres. Stell and Weis 33] calculate the initial susceptibility of DHS using this modification and a further improved version. They get larger values for $\chi$ that agree better with the Monte-Carlo data.

Patey [34] improves the theory of Wertheim by retaining the ansatz (3.19) but expanding the full relation (3.16) linear in $f_{D}$ and $f_{\Delta}$. This approach is known as linear HNC (LHNC), although it was pointed out later that an equivalent theory was proposed already in 1973 by Wertheim [35] himself, called single-superchain theory. In contrast to the MSA, the LHNC requires a numerical calculation of the correlation functions $h_{S}(r), h_{D}(r)$, etc. to find the initial susceptibility. Within the LHNC $\chi$ depends on both $\phi$ and $\lambda$ independently. Patey did not apply the "pure" HNC but uses again $h_{S}$ and $c_{S}$ from the system of nonpolar hard spheres as system of reference (known as reference LHNC or RLHNC).

The RLHNC was followed by the reference quadratic HNC (RQHNC) proposed by Patey, Levesque and Weis. In [36] this approach is applied to DHS. In the RQHNC (3.16) is expanded up to quadratic terms in $f_{D}$ and $f_{\Delta}$. Differences in $\chi$ between RQHNC and 
RLHNC amount only up to a few percent for $\lambda<2$ and relatively large $\phi \approx 0.4$. But they are more significant for larger $\lambda$. However, later on it turned out in Monte-Carlo simulations (see Sec. IIIC) that both theories overestimate $\chi$ here - the apparently better RQHNC even more so than the RLHNC.

These authors considered also Stockmayer particles, using DHS as a reference system. They argue that a Stockmayer system with $\phi=0.8 \pi / 6$ and $1 / v_{0}=1.35$ should be similar to a system of DHS with the same $\phi$ for not too large $\lambda$. Indeed, the results for the susceptibility do not differ very much for $\lambda<2$. For higher coupling constants the RQHNC now gives smaller values for $\chi$ than the RLHNC.

Agrafonov, Martinov and Sarkisov [37] expand the full HNC equation in terms of $\lambda$ and obtain the result

$$
\frac{\chi^{2}}{\chi+1}=64 \phi^{2} \lambda^{2}\left(1-\frac{8}{3} \phi \lambda\right)
$$

for DHS. This expression yields after solving for $\chi$ the correct terms in $\phi \lambda$ and $\phi^{2} \lambda^{2}$. However, the next term is already incorrect. Chan and Walker [38] propose to expand the HNC approximation (3.16) given in the form

$$
g(1,2)=\exp \left[h(1,2)-c(1,2)-v_{12}\right]
$$

in terms of rotational invariants for the orientational distribution of $\hat{\mathbf{m}}_{1}$ and $\hat{\mathbf{m}}_{2}$, truncating the expansion after a sufficient number of terms. They take into account however only three terms, namely the functions already used in the MSA, RLHNC, and RQHNC. Using this truncated HNC ansatz (THNC) they obtain even higher values for the susceptibility of DHS than predicted by the RQHNC.

In 1985 Fries and Patey [39] finally extend the ansatz (3.19) by adding more terms. They also use (3.16) in a form differentiated with respect to $r_{12}$ eliminating the logarithmic term. Solving numerically the full RHNC approximation for DHS in that way they come to results for $\chi$ that are in much better agreement with Monte-Carlo calculations. In the same year Lee, Fries, and Patey [40] also investigate the Stockmayer system. As in the case of DHS the results agree better with numerical data than those of RLHNC and RQHNC. Finally, in 1986, Fries and Patey 41] apply the similar Percus-Yevick approximation to DHS finding it less well suited to predict the susceptibility than the RHNC and even the MSA.

Another modification is done by Lado [42], who varies the diameter of the nonpolar hard spheres in the system of reference to minimize the free energy. Lomba, Martin and Lom- 
bardero [43] solve the pure HNC finding for the correlation functions even better agreement with Monte-Carlo calculations than for the RHNC.

\section{Numerical simulations}

Numerical simulations for calculating the susceptibility are mostly based on Monte-Carlo methods. $\chi$ is either determined from the magnetization fluctuations $\left\langle M^{2}\right\rangle$ in the absence of an external field or more directly from the magnetization in small fields. Calculating the susceptibility from the simulation results for $h(1,2)$ are less appropriate since $\chi$ depends on the long-range behavior of $h(1,2)$ that cannot be determined accurately in finite simulation cells [36].

The influence of the distant dipoles can be incorporated by cutting off the dipolar interaction at a finite distance $R_{s}$ and replacing the dipoles beyond $R_{s}$ by a magnetic continuum of given $\chi$ (reaction field method). This susceptibility is either adjusted to the susceptibility of the simulated system or set to an arbitrary value (commonly to $\chi=0$ ). The chosen value then enters into the determination of the system's susceptibility.

Another method that is the most commonly used today is the Ewald summation based on a periodic continuation of the system together with an effective method for calculating the resulting total dipolar field. A discussion of the different methods can be found e. g. in [44, 45]. The Ewald summation is described in [46].

Monte-Carlo calculations of the free energy and other thermodynamical quantities that depend less strongly on the long-range nature of the dipolar forces were already performed in the seventies of the last century [32, 47, 48, 49]. Patey et al. [34, 36, 50] calculate the correlation function $h(1,2)$ and compare with the predictions of the LHNC and QHNC. An indirect comparison for the susceptibility, demonstrating the superiority of the MSA over the Onsager model can be found already in [49].

An early Monte-Carlo calculation of $\chi$ is done by Adams and McDonald [46] in 1976. The authors however do not consider a fluid but f. c. c. and s. c. lattices. They compare Ewald sum and reaction field results for interaction strengths up to $\phi \lambda \approx 2$. Levesque, Patey, and Weis [50] calculate $\chi$ for $\phi=0.8 \pi / 6$ and $\lambda \leq 1$. They compare different methods and system sizes and in 1982 they also study the case $\lambda=2$ [51]. Adams [44] considers already in $1980 \lambda=2.75$. Lado [52] uses a combination of Monte-Carlo calculation and 
RHNC for the long-range part of the potential.

Susceptibility calculations for Stockmayer particles were mainly performed for the values $\phi=0.8 \pi / 6$ and $v_{0}=1 / 1.35$ as already considered by Patey, Levesque and Weis [36]. Pollock and Alder [53] consider values for $\lambda$ up to 3 while Adams and Adams [45] study different values for $v_{0}$. Levesque and Weis [54] calculate $\chi$ for $\lambda=2$, and Evans and Morriss [55] do so for $\lambda \leq 1.7$. Neumann, Steinhauser, and Pawley [56] consider the parameter combination $v_{0}=1 / 1.15, \phi \approx 0.43$, and $\lambda=2.6$. Hesse-Bezot, Bossis, and Brot [57] use in 1984 molecular dynamic methods to investigate $\lambda=2$.

Some of the works cited above treat the long-range nature of the dipolar interaction incorrectly, see the discussion by Neumann [58], Neumann and Steinhauser [59, 60], and Gray et al. [61]. These papers also discuss the relationship between the reaction field and the Ewald sum approach. Further discussions of the Ewald summation and of other techniques used for dipolar systems were given recently by Wang, Holm, and Müller [62, 63].

\section{A comparison}

Unfortunately most of the older works deal with systems with large volume fractions: a typical value is $\phi=0.8 \pi / 6=0.419$. This is reasonable for customary polar liquids but it is very high for ferrofluids. Fig. 4 shows a comparison of the most important theories discussed above for the case of DHS. In this figure $\chi$ is plotted as a function of $\lambda$ for fixed $\phi=0.419$. The Monte-Carlo data (denoted by circles) and the results for RLHNC and RHNC were taken from [39] and a similar plot in [28]. Onsager theory and MSA clearly underestimate the Monte-Carlo data while Weiss theory and RLHNC overestimate them. The RHNC works much better, as does the numerically much less complicated result by Tani et al. [26]. The curve denoted as "Huke and Lücke" refers to a cluster expansion theory 64] for the full magnetization curve. In the linear case considered here in this section this theory [64] yields Eq. (3.8) and in addition also the $\phi^{2} \lambda^{n}$ terms given by Eq. (3.11).

The large scatter of the Monte-Carlo results for $\lambda=2.75$ is indicated in Fig. 4 as well. Surprisingly there seems to be no final conclusion in the literature concerning the appropriate value of $\chi$ for $\lambda=2.75$ despite the fact that these different Monte-Carlo results have been in the literature for quite a while. The different predictions for $\chi$ at $\lambda=2.75$ are discussed in [60] where preference is given to a relatively low value of $\chi \approx 64$. 


\section{E. Other potentials}

The susceptibility of some variants of the DHS and Stockmayer particle systems were also investigated. The system of dipolar sticky hard spheres, where the short-range potential contains an additional $\delta$-function term, were investigated by Chapela and Martina [65] using integral theories like MSA and RLHNC to calculate $\chi$. The authors come to contradictory results concerning the effect of the additional term in the potential on $\chi$. Joslin and Gray 66. show that the second dielectric virial coefficient of dipolar sticky hard spheres is positive.

Kusalik [67, 68, 69] investigates in 1989 and the following years dipolar soft spheres, i. e. Stockmayer particles without the attractive $r^{-6}$-term in the short-range part of the potential using the RLHNC and the RHNC as well as Monte-Carlo calculations. The Monte-Carlo results for $v_{0}=1 / 1.35, \phi=0.8 \pi / 6$, and $\lambda=2$ are very similar to those for Stockmayer particles. But RLHNC and RHNC overestimate $\chi$ somewhat more.

Henderson, Boda, Szalai, and Chan [70, 71] apply the MSA, the theory by Tani et al. [26], and Monte-Carlo calculations to dipolar Yukawa particles with a hard core. Again, the results are found to be similar to those for dipolar hard spheres and Stockmayer particles. The Monte-Carlo data for $\chi$ at $\phi=0.8 \pi / 6$ and $\lambda=1,2$ depend little on the strength of the Yukawa potential and they agree well with the Monte-Carlo data for dipolar hard spheres. The perturbation theory by Tani et al. is found to reproduce the Monte-Carlo data better than the MSA.

To summarize, the nature of the short-range interaction seems to have a rather weak influence on the susceptibility, even for relatively high densities.

\section{F. Polydisperse theories}

In real ferrofluids the influence of polydispersity can normally not be neglected. In the case of ideal paramagnetism discussed in Sec. IA the initial susceptibility is given by

$$
\chi_{L}=\sum_{i} \frac{m_{i}^{2}}{3 \mu_{0} V k_{\mathrm{B}} T},
$$

as follows from (2.5). Therefore $\chi_{L}$ is proportional to the second moment of the distribution of the magnetic moments and larger than in the monodisperse case if the average particle volume is kept constant. When particle interactions are taken into account even higher moments of the distribution enter into the initial susceptibility. 
Several of the theories discussed above were also extended to the system of polydisperse or at least bidisperse DHS, where the particles have different hard sphere diameters and/or carry different magnetic moments. Already in 1973 the MSA of Wertheim is applied to the polydisperse case by Adelman and Deutch [72], although restricted to particles with a common diameter. The authors define an equivalent monodisperse system having the same thermodynamical properties.

Isbister and Bearman [3] generalize the MSA to mixtures with arbitrary diameters. The susceptibility predicted by this theory is calculated by Freasier, Hamer, and Isbister [4]]. The authors also describe a comparable monodisperse system giving at least approximately the same results. Ramshaw and Hamer simplify the evaluation [75]. Cummings and Blum 76. compare results of the MSA to Monte-Carlo results for the bidisperse case.

Lee and Ladanyi present [77] a RLHNC extension and generalizations [78] of the RHNC and the cluster expansion by Tani et al. [26]. They compare to the Monte-Carlo data from [76], coming to similar conclusions about the quality of the different theories as in the monodisperse case discussed above (Sec. IIID).

\section{G. Applications to ferrofluids}

With the preparation of highly concentrated ferrofluids real physical systems became available that have much more in common with the simple model system of DHS than the ordinary polar fluids. Ferrofluids thus allowed not only more reliable experimental tests but they also initiated new theoretical investigations.

Two-dimensional Monte-Carlo simulations on the initial susceptibility of ferrofluids are performed by Menear, O'Grady, Bradbury et al. in the early eighties [79, 80, 81, 82, 83]. Here the particles are not modeled as pure hard spheres but the sterical repulsion of the surfactants according to Rosensweig (2.15) is also taken into account. In [83] a polydisperse ferrofluid is simulated. In particular, the temperature dependence of the susceptibility entering via $\lambda \sim 1 / T$ is investigated. A Curie-Weiss law $1 / \chi \sim T-T_{0}$ is found as to be expected from simple mean field models, e. g. the Weiss model.

Bradbury, Martin, and Chantrell [84, 85] also perform full three-dimensional simulations for DHS with additional sterical repulsion and obtain good agreement with RHNC calculations. The authors again find a Curie-Weiss law for $\chi(T)$ but less pronounced than for the 
2D calculations. The calculations are done for a small $\phi=0.01$ and very high $\lambda$ up to 7.21. These values refer to the real hard sphere diameter. However, the dipolar coupling would be better characterized by using the total diameter of the particles, i.e., of core plus polymer layer. That would reduce $\lambda$ to values of maximal 2.63. In this $\lambda$-range much simpler cluster expansion theories still work and reproduce the result. It should be mentioned that although $1 / \chi=c\left(T-T_{0}\right)$ is a very good approximation in that range, the Weiss model already fails since it predicts significantly different values for $c$ and $T_{0}$.

A behavior of $1 / \chi \sim T-T_{0}$ is also found in experiments [79, 86]. The results in [9] however rely on data in a narrow range of temperatures of $60 \mathrm{~K}$ where only very strongly interacting ferrofluids should exhibit a pronounced nonlinear behavior. In 86] a negative value for $T_{0}$ is found, i. e., a Néel behavior that was not predicted by the theories. A more comprehensive experimental investigation of the initial susceptibility is published in 1990 by Holmes, O'Grady, and Popplewell [87]. Inspecting a broader range of temperatures the authors show deviations from the Curie-Weiss law for ferrofluids based on magnetite. They also argue that the Néel behavior found in [86] may result from measurements for too large fields, i. e. already in the nonlinear range of the magnetization curve. They find a linear and a quadratic term in the concentration dependence of the susceptibility. The nonlinear behavior of $1 / \chi(T)$ is confirmed by Williams et al. [88] in 1993.

Morozov et al. [89, 90] apply the MSA to ferrofluids. Further results can be found in [91]. The authors demonstrate good agreement with experiments concerning the concentration and temperature dependence of $\chi$. As discussed above in Sec. IIID the MSA does not seem to be a good theory for strongly interacting systems. The investigated ferrofluids $(\phi \leq 0.172$, $\chi<10)$ however fall in range where the difference in $\chi$ between MSA and cluster expansions [26, 64] is only a few percent. Applying the formula (3.8) shows that the $\chi_{L}^{3}$-term which is correctly predicted by the MSA gives only minor contributions to $\chi$.

Pshenichnikov [93] compares several theories, namely the Onsager model, the Weiss model, the MSA and a theory by Ivanov et al. 94] with experimental data in a similar range of $\phi$ and $\lambda$. The latter theory [94] deals with the equilibrium magnetization for arbitrary fields. It is explained in the next section. For the initial susceptibility this theory gives $\chi=\chi_{L}+\chi_{L}^{2} / 3$, i. e., Eq. (3.8) up to second order. Pshenichnikov shows good agreement with both Ivanov's theory and the MSA, thereby also indirectly demonstrating the small influence of the $\chi_{L}^{3}$-term that is present in the MSA but not in Ivanov's theory. Both 
theories however fail to adequately describe the temperature dependence of ferrofluids with $\chi(T)$ lying in the range between $\approx 20$ and 80 .

Kalikmanov 92] proposes in 1999 the so-called algebraic perturbation theory that is corrected by Szalai, Chan and Henderson [95] and found to be identical to the cluster expansion theory of Tani et al. [26].

Van Ewijk, Vroege, and Philipse [18] perform susceptibility measurements on highly concentrated ferrofluids and compare with different theories. For a ferrofluid with $\lambda \approx 2.7$ and $\chi_{L} \leq 6$, they find a surprisingly low susceptibility of $\chi \leq 10$, best described by the Onsager theory or the MSA. These results seem to be in contradiction to the data presented in Fig. 4, although $\phi$ is slightly higher there. Some theoretical support to these findings can however be found in a paper by Pshenichnikov and Mekhonoshin [96], who present in 2000 Monte Carlo data in the same range of $\chi_{L}$. For $\lambda=3$ and $\lambda=4$, they find similar low values for the susceptibility. They explain the low susceptibility with aggregates of particles, that have a small combined magnetic moment and interact with the field only weakly. 


\section{MAGNETIZATION}

The equilibrium magnetization of a ferrofluid cannot be described in general by a simple Langevin ansatz or its polydisperse generalization. Experimental hints to this fact can already be found in a paper from 1979 by Tari et al. [97] where a comparison of a magnetization curve with a theoretical curve based on an independently measured size distribution is made. However, the theories for the magnetization of, say, dipolar hard spheres in arbitrary fields are less advanced than for the special case of the initial susceptibility. Most of the theories discussed below have been devised with regard to the application to ferrofluids. For this reason, many of them were directly or shortly after extended to include also the effects of polydispersity.

When comparing a polydisperse ferrofluid with a monodisperse one with the same average magnetic moment of the particles and the same volume fraction, the equilibrium magnetization curve will be steeper for small fields in the polydisperse case since the initial susceptibility is higher. The asymptotic behavior for high fields will however be the same. This results in a maximal difference between the magnetization curves for medium fields $\alpha \approx 1$.

Pollock and Alder [53] and Adams and Adams [45] perform Monte-Carlo calculations for the equilibrium magnetization of Stockmayer particles. In 1981 Høye and Stell [98] propose a mean field model based on general thermodynamic considerations and compare their results with the data from [53]. The magnetization in this model is given by

$$
M=M_{\text {sat }} \mathcal{L}\left(\frac{m}{k_{\mathrm{B}} T} H_{\mathrm{eff}}\right),
$$

where $H_{\text {eff }}$ is an effective field

$$
H_{\mathrm{eff}}=H+\frac{1}{3}(1-\Theta) M
$$

Here $\Theta$ is to be taken from the results for the initial susceptibility. For $\Theta=0$ the formula reduces to that of the Weiss theory. Dikanskii [99] proposes a concentration dependent term for an effective field calculated from a fit to susceptibility data. Sano and Doi [100] investigate phase transitions in ferrofluids using a model of a randomly filled cubic lattice. As an additional result they obtain the Weiss expression for the equilibrium magnetization.

Woodward and Nordholm [101] propose an effective potential for the dipolar interaction 
that results from averaging over the orientations of the dipoles according to

$$
\exp \left(-V_{12}^{\mathrm{eff}} / k_{\mathrm{B}} T\right)=\left\langle\exp \left(-V_{12}^{D D} / k_{\mathrm{B}} T\right)\right\rangle_{\hat{\mathbf{m}}_{1}, \hat{\mathbf{m}}_{2}}
$$

The so defined $V^{\text {eff }}$ is then a function of particle separation and temperature. It vanishes for large distances as $r_{12}^{-6}$. In 1986 [102] these authors extend their ansatz to a theory for the equilibrium magnetization. They propose a functional for the free energy $F$ of a magnetic continuum in a magnetic field. Minimized with respect to the orientational distribution of the dipoles it gives for zero field an expression for $F$ that contains $V^{\text {eff }}$ as interaction potential. Including the external field the dipolar interaction is described by a long- range part as in the Weiss model and a short-range part that is a generalization of $V^{\text {eff }}$ which, however, depends also on the magnetic field. The magnetization has the form (4.14.2) with a function $\Theta(M, T)$ that can be calculated numerically. The authors compare the resulting initial susceptibility with the RLHNC predictions. The results are however not convincing: They are larger than in the RLHNC already for $\phi \approx 0.3$ and diverge for $\phi \approx 0.4$. Comparisons for DHS with the magnetization results of Høye and Stell can be found in [103].

Berkovsky, Kalikmanov, and Filinov [13, 104, 105] develop between 1985 and 1992 a thermodynamical theory for ferrofluids. Based on cluster expansion methods they calculate the magnetization in two special cases. They derive expressions for spherical geometries where $H=H_{e}-M / 3$. For small fields $\alpha \ll 1$ the authors obtain for the susceptibility with respect to the external field $H_{e}$

$$
\chi_{e}=\frac{\partial M}{\partial H_{e}}=8 \lambda \phi-\frac{160}{3} \lambda^{3} \phi^{3} .
$$

If one restates this expression into a result for $\chi$ a missing $\phi^{2} \lambda^{2}$-term appears and one obtains (3.8). For stronger fields they get

$$
M=M_{\mathrm{sat}}\left[\mathcal{L}\left(\alpha_{e}\right)+L_{1,2}\left(\alpha_{e}\right) \phi \lambda^{2} f(\phi)\right]
$$

Here $L_{1,2}\left(\alpha_{e}\right)$ is an analytic expression and $f(\phi)$ depends on the correlation function $g(1,2)$ of nonpolar hard spheres that is used as reference system. Again, by restating the expression in a form $M=M(H)$ an additional term of order $\phi \lambda$ shows up. Here one has to note that $M / M_{\text {sat }}$ is independent of $\phi$ and $\lambda$ in leading order, whereas $\chi$ starts with a $O(\phi \lambda)$-term. Likewise, terms of order $\phi^{m} \lambda^{n}$ in $M / M_{\text {sat }}$ always correspond to $O\left(\phi^{m+1} \lambda^{n+1}\right)$-terms in $\chi$. 
Between 1990 and 1992 also Ivanov et al. 94, 106, 107, 108, 109] develop a thermodynamical theory of ferrofluids also using cluster expansion techniques. For a needle-shaped geometry in which $H_{e}=H$ they obtain the correct result in $O(\phi \lambda)$

$$
M=M_{\text {sat }}\left[\mathcal{L}(\alpha)+8 \phi \lambda \mathcal{L}(\alpha) \mathcal{L}^{\prime}(\alpha)\right]
$$

A correct polydisperse generalization, replacing $\mathcal{L}(\alpha)$ by $\mathcal{L}^{\text {poly }}(\bar{\alpha})(2.7)$, is also given.

Lebedev [110] suggests to apply the MSA also to finite magnetic fields. To that end he replaces the equations (3.22) by

$$
M(H)=\frac{M_{L}(H)}{q(-x)}, \quad \frac{\mathrm{d} M(H)}{\mathrm{d} H}=\frac{q(2 x)-q(-x)}{q(-x)},
$$

with $q(x)$ given as before by the third equation in (3.22). Thus, $\chi$ and $\chi_{L}$ in the first equation of (3.22) is replaced in (4.7) by $M$ and $M_{L}$, respectively. The second equation of (4.7) can be derived by combining the first two equations in (3.22) and replacing the initial susceptibility $\chi$ by the susceptibility $\mathrm{d} M(H) / \mathrm{d} H$ for finite fields. Hence, the equations (4.7) reduce to the MSA for small fields.

Morozov et al. [91, 111] present in 1990 a real extension of the MSA aimed at predicting the magnetization for arbitrary fields. They use the so-called Lovett-Mou-Buff-Gubbins equation [112, 113] to relate the one-particle probability density $\rho(\mathbf{r}, \mathbf{m})$ in the presence of an external field to the direct correlation function $c(1,2)$ taken in the MSA form of Eq. (3.18). With Wertheim's method [29] they then derive from the Ornstein-Zernike equation two independent Percus-Yevick like equations for a hard-sphere fluid with renormalized densities. As a result of this approximation scheme they present $M$ given by the Langevin magnetization for an effective field $C \alpha$

$$
M=M_{\text {sat }} \mathcal{L}(C \alpha)
$$

Here $C$ has to be calculated as follows: Let

$$
A=\frac{\partial \ln M}{\partial \ln (C H)} \quad \text { and } \quad 35 x^{2}-5(10-3 A) x-7(7+3 A)=0
$$

With $x_{1}$ and $x_{2}$ being two solutions of the quadratic equations, $C$ must be given selfconsistently by

$$
C=\left[1+\frac{4 \pi}{3}\left(1+x_{1}\right) \frac{M}{H}\right] \frac{1}{q\left(x_{1} y\right)}=\left[1+\frac{4 \pi}{3}\left(1+x_{2}\right) \frac{M}{H}\right] \frac{1}{q\left(x_{2} y\right)}
$$


Here $y$ is defined via the second equality and $q(x)$ is still given by the expression in (3.22). The authors also propose a polydispersive generalization. Comparisons with experiments can be found in [91, 111]: The MSA reproduces well the magnetization curves of polydisperse ferrofluids with saturation magnetizations up to $87 \mathrm{kA} / \mathrm{m}$.

Based on preliminary work 114] concerning the 2D case Bradbury and Martin [115] and independently Ayoub et al. [116] develop the so-called dimer model of dipolar interaction. The partition function for $N$ particles is written as the $(N / 2)$ th power of a two-particle partition function calculated via a cluster expansion assuming that the two particles are closer than a typical next-neighbor distance. This model has several shortcomings. The long-range character of the forces is not taken into account leading to an expression for $M$ in which the $\phi \lambda$-term is missing. Also the calculated $O\left(\phi \lambda^{2}\right)$-terms are wrong in both cases and moreover they do not agree with each other [117]. Even the corrected calculation leads to a wrong result by a constant factor due to the somewhat arbitrary assumption of using the next-neighbor distance as a cutoff.

Zubarev and Iskakova [118] present a magnetization equation relying on a model of noninteracting magnetic clusters (chains). They derive the equilibrium distribution of chain lengths based on a simple expression for the free energy of a system of such chains. The magnetization is then formally the equilibrium magnetization of a polydisperse ideal paramagnetic gas where the chains are the fundamental constituents. The chain length distribution is however a function of the magnetic field and furthermore of $\phi$ and $\lambda$. Abu-Aljarayesh and Migdadi [119] investigate this theory further, calculating the entropy and some equilibrium magnetization curves.

Pshenichnikov, Mekhonoshin and Lebedev [120] suggest an equation for the equilibrium magnetization that reads

$$
M=M_{\text {sat }} \mathcal{L}\left[\alpha+\frac{m M_{\text {sat }}}{3 k_{\mathrm{B}} T} \mathcal{L}(\alpha)\right]
$$

It agrees better with their experiments in the range of medium fields than the expression (4.6) that gives the same initial susceptibility. It furthermore gives more consistent results for the diameter distribution of polydisperse ferrofluids at different stages of dilution. Furthermore, their earlier result (4.6) can be derived from Eq. (4.11) by expanding the latter up to first order in $\phi$ and $\lambda$ since $m M_{\text {sat }} / 3 k_{\mathrm{B}} T=8 \phi \lambda$. On the other hand, Eq. (4.11) can be obtained from the Weiss formula (2.8) by iterating and stopping after the first step. How- 
ever, Eq. (4.11) has the advantage over Eq. (4.6) that it always produces physically sound magnetization curves with $M$ being a monotonic function of $H$ with $0 \leq M<M_{\text {sat }}$. This is not the case for Eq. (4.6) when $\phi \lambda$ becomes too large.

In 2000 the authors of this review [64] perform a cluster expansion calculation to derive some of the terms in the sum

$$
M=M_{\text {sat }}\left[\mathcal{L}(\alpha)+\sum_{a, b} L_{a, b}(\alpha) \phi^{a} \lambda^{b}\right]
$$

namely a number of $L_{1, b}$-terms and the leading term, $L_{2,2}$, in $O\left(\phi^{2}\right)$. All the calculated terms are given as analytic expressions. The first one, $L_{1,1}(\alpha)=8 \mathcal{L}(\alpha) \mathcal{L}^{\prime}(\alpha)$, yields the result (4.6) of Ivanov et al. and $L_{1,2}$ is the same as in the expression (4.5). A generalization to the polydisperse case is given in [121].

In the same year, Klapp and Patey [122] present results obtained from two different integral equation approximation schemes that use either the MSA or the RHNC as twoparticle closures. The singlet density $\rho$ and the external field is related in formally exact ways either via the first BGY hierarchy equations 28] to the pair correlation function $g(1,2)$ or via the Lovett-Mou-Buff-Wertheim equations [112, 123] to the direct correlation function $c(1,2)$. The results turn out to be strongly dependent on the form of the closure used. For example, the authors find in zero field ferromagnetic behavior using the RHNC closure that is absent in case of the MSA closure [122].

Pshenichnikov and Mekhonoshin [96] present Monte-Carlo results for a finite system giving $\chi$ as a function of $\chi_{L}$ for fixed $\lambda=1,3$, and 4 . They also calculate the equilibrium magnetization for a volume fraction $\phi=0.226$ and $\lambda=1$ and 4 . The results for $\lambda=1$ can quite well described by the equation (4.11). The equilibrium magnetization for $\lambda=4$ is however smaller than predicted by any theory for interacting particles and is explained as result of the formation of clusters with a small total magnetic moment.

Ivanov and Kuznetsova [124] perform a cluster expansion calculating the $O\left(\phi^{2} \lambda^{2}\right)$-term in $M / M_{\text {sat. }}$. While their results are not given explicitly they seem to reproduce [125] the result of [64]. The authors of 124] suggest an extension of Eq. (4.11) that reads

$$
M=M_{\text {sat }} \mathcal{L}\left(\frac{m H_{\text {eff }}}{k_{\mathrm{B}} T}\right), \quad H_{\text {eff }}=H+\frac{1}{3} M_{\text {sat }} \mathcal{L}(\alpha)+\frac{1}{144} M_{\text {sat }}^{2} \mathcal{L}(\alpha) \frac{\mathrm{d}}{\mathrm{d} H} \mathcal{L}(\alpha) .
$$

A polydisperse generalization, replacing $\mathcal{L}$ by $\mathcal{L}_{\text {poly }}$ is also given. The formula (4.13) produces sound magnetization curves for arbitrary $\phi, \lambda$ as in the case of Eq. (4.11). When comparing 
the results of [124] with those of the cluster expansion [64] one has to be aware that in [124] some calculated terms that do not contribute to the initial susceptibility were discarded in order to be able to write the result in the form (4.13). Thus, when this equation is expanded in $\phi \lambda$ one does not recover the general $O\left(\phi^{2} \lambda^{2}\right)$-term of (4.12) while it is reproduced in the expression for the initial susceptibility.

Molecular dynamics simulations of monodisperse dipolar systems are performed in 2002 by Wang, Holm, and Müller [126] for several combinations of $\phi$ and $\lambda$. They use a modified Stockmayer potential with a shifted and truncated Lennard-Jones potential. Comparing with the theory of Ivanov and Kuznetsova [124] they find good agreement for $\lambda \leq 2$ but deviations for higher $\lambda$, both for the magnetization curve and the initial susceptibility. The authors also demonstrate that $M$ and $\chi$ are not functions of the product $\phi \lambda$ alone as predicted in [124] and that the theory 124] becomes less applicable for large $\lambda$ and small $\phi$. Furthermore they analyze the microstructure of the simulated fluid and determine the length distribution of chains. They show that the susceptibility can be better explained by replacing the Langevin susceptibility in (3.8) by the susceptibility of a system of ideal dipolar chains with a length distribution found in the simulations. In 2004 Ivanov, Wang, and Holm [127] use a variant of the chain formation model of [118] to obtain a length distribution independent of the simulation results. Already in 2003 Wang and Holm publish simulation results on bidisperse dipolar systems [128]. For a system containing particles with $\lambda=1.3$ and $\lambda=5.32$ and a total volume fraction of $\phi=0.07$ they report that the theory of Ivanov and Kuznetsova [124] works well for volume fractions $\phi_{L} \leq 0.02$ of the large particles. However, differences become apparent for larger fractions of the large particles, say, $\phi_{L}=0.05$ and even more so in the limiting case, $\phi_{L}=0.07$, without any small ones, i.e., for a monodisperse fluid containing only large particles. Kristóf and Szalai [129] perform in the same year Monte-Carlo simulations for two polydisperse ferrofluids with realistically chosen parameters. They find good agreement with the polydisperse version of (4.6).

Raşa et al. [130] measure in 2002 the initial susceptibility and the magnetization of ferrofluids at various stages of dilution. The susceptibility is found to be best described by Ivanov's theory [94] and the MSA [111]. 


\section{NONSPHERICAL POTENTIALS AND HIGHER-ORDER MAGNETIC MO- MENTS}

Real ferrofluid particles can be expected to have small deviations from a perfectly spherical shape. Such deviations affect not only the form of the nonmagnetic part of the interparticle potential. Also the magnetic interaction potential will be changed since the magnetic field of a nonspherical magnetized particle will contain also higher magnetic moments beyond the dipolar one. Both features will have some influence on the susceptibility and the magnetization. The susceptibility of systems of simple nonspherical dipolar particles and particles with dipolar and quadrupolar particles where also investigated on some occasions.

A straightforward generalization of the system of DHS is the system of dipolar hard ellipsoids. Joslin calculates 131] numerically the second dielectric virial coefficient for this system for different $\lambda$ and axis ratios. For spheres, this coefficient is positive, see Eq. (3.11). However, it depends strongly on the axis ratio: it grows with growing prolateness but it becomes negative already for only slightly oblate particles. Perera and Patey [132] investigate this system by means of the HNC, finding the same trend.

Dimer ferrofluid particles were modeled by dipolar fused spheres (dumbbells). A MonteCarlo simulation of Stockmayer dimers can be found in a paper of de Leeuw and Quirke [133]. $\chi$ is found to decrease with growing sphere separation. Dimers of dipolar hard spheres are simulated by Lomba, Lombardero, and Abascal [134]. They study the density dependence of the susceptibility for fixed dipolar strength and find a decrease of $\chi$ at high densities due to the hindrance of particle rotation.

Dipolar particles of spherical shape that carry, however, an additional quadrupolar moment are investigated with respect to their susceptibility by Patey, Levesque, and Weis [135] and Carnie and Patey [136] for different choices of the quadrupolar tensor using integral theories. The susceptibility is shown to decrease with growing quadrupolar moment.

In view of the fact that the average shape of a ferrofluid particle is roughly spherical it seems to us that systems of identical nonspherical or multipolar particles that have been considered in the literature are probably less applicable to describe real ferrofluids than systems of DHS or Stockmayer particles. To improve upon monodisperse models of identical particles one would have to consider at least binary mixtures of particles of different nature, e. g. of prolate and oblate ellipsoids or of monomers and dimers. 


\section{PHASE TRANSITIONS}

Two different kinds of phase transitions are addressed in this section. The first one is the condensation of magnetic particles into a denser phase. The second one is the transition from an isotropic nonmagnetic phase to a state with spontaneous long-range magnetic order.

There are other, less intensively investigated transitions in systems of dipolar particles such as fluid/solid transitions 137, 138, 139] or the demixing of particle species in bidisperse systems [140]. Another example of a phase transition that we do not touch here is the emergence of a dipole glass state at low temperatures for systems of dipoles in a solid matrix, like frozen ferrofluids [141, 142, 143]. The existence of such a state in liquid ferrofluids has been reported, e. g., in [144]. But then it was shown that the observed temperature behavior of the susceptibility can be explained as an effect of the temperature dependence of the viscosity of the carrier liquid [145]. A review of this subject for the electrical case can be found in [146].

\section{A. Dilute/dense phase transitions}

Ferrofluid particles possess an isotropic attractive potential: the outer tail of the van der Waals attraction that is not shielded by the steric or electrostatic repulsion. This can result in the creation of small clusters, as seen by Donselaar et al. in cryo-TEM images [147].

When the attractive potential has a strength of about $k_{\mathrm{B}} T$ a dilute/dense phase transition should occur. It is possible to weaken the repulsive shielding of the ferrofluid particles enough to allow such a reversible phase transition without reducing it so much that an irreversible agglomeration takes place as observed in experiments with ionic ferrofluids 148, 149, 150, 151]. This reversible transition is a kind of gas/liquid transition. It affects however only the magnetic subsystem of the ferrofluid - the carrier system remains always liquid. We will use the term "dilute/dense" instead of "gas/liquid" phase transition also when referring to the common model systems without any carrier liquid to avoid a confusion of terms. The dense phase exists in the form of (mesoscopic) droplets.

In a typical ferrofluid the isotropic interaction is rather weak. In Fig. 3, for example, the best fitting Lennard-Jones potential has a depth of $v_{0}=0.16$. Since a dilute/dense transition

does not occur in pure Lennard-Jones systems when $v_{0}<0.76$ [152] one concludes that such 
a phase transition might possibly occur in ferrofluids with smaller $v_{0}$ only if the dipolar interactions have a net supporting, i.e., attractive effect.

A dilute/dense transition observed in experiment that might be caused mainly by dipolar interactions was reported by Mamiya, Nakatani, and Furubayashi [19]. These authors investigate a ferrofluid consisting of iron-nitride in kerosene with $3 \lesssim \lambda \lesssim 10$. They find a hysteresis in $\chi$ as a function of temperature when $\phi$ is roughly between 0.002 and 0.06 . For larger $\phi$ the hysteresis vanishes. Furthermore, $\chi(T)$ is larger when cooled than when reheated in the hysteresis region. The authors explain this with the presence of closed rings or drops of particles with mostly vanishing total magnetic moment in the low-temperature phase.

When studying phase transitions of dipolar hard spheres, the dipolar coupling constant $\lambda \sim 1 / T$ serves as the dimensionless inverse temperature. Likewise, $\phi$ serves as dimensionless density. In the case of Stockmayer particles both, $\lambda$ and $v_{0}$, provide an inverse temperature scale and in general the latter is used. The other quantity or the temperature independent quantity $\lambda / v_{0}$ serves as an additional parameter.

Considering these main model potentials it is clear that a system of Stockmayer particles will exhibit a dilute/dense phase transition below some critical $T_{c}$ since it contains the Lennard--Jones potential as special case. The additional dipole-dipole interaction raises $T_{c}$ (see below) an effect that cannot be explained trivially, since this interaction can be both repulsive and attractive and its orientational mean is zero. However, an averaging procedure as in (4.3) that takes into account the fact that attracting configurations are preferred yields an attracting mean dipolar contribution.

In the case of dipolar hard spheres and also soft spheres it is even more unclear a priori whether such systems show a dilute/dense phase transition since here the dipolar interaction is the only one that could cause such a behavior. As we will discuss below, the question whether this phase transition exists or not in pure dipolar hard sphere systems is still being debated.

In systems of Stockmayer particles with small $v_{0}$ strong dipolar couplings seem to be necessary in order to raise the critical temperature enough to observe a dilute/dense transition. For example, Frodl and Dietrich [153] find using a density-functional theory that already a dipolar coupling constant of $\lambda \approx 1.5$ is necessary to lower the critical point to about $v_{0} \approx 0.4$. According to Monte-Carlo calculations by Hendriks, van Leeuwen et al. [154, 155, 156] be- 
tween 1989 and 1994 it is $\lambda \approx 2.35$ for $v_{0} \approx 0.4$, and Monte-Carlo calculations of Stevens and Grest [157] from 1995 give a very similar value here. That means that common ferrofluid particles with small $v_{0}$ will not show a dilute/dense phase transition within a reasonable temperature range except in systems where the dipolar interaction is the by far dominating contribution. It may seem that in such a case the short-range attraction should play no more any role and that Stockmayer particles with high $\lambda$ should behave similar to dipolar hard spheres. However, as mentioned already this does not seem to be the case.

In the 1970s and 80s the dilute/dense phase transition of dipolar hard spheres was studied by using mainly analytical and semianalytical theories that were conceived to investigate the equilibrium magnetization or initial susceptibility. A usual kind of $(V, T)$-phase diagram was found with a roughly parabolic coexistence curve and no phase transition above some critical $T_{c}$ (i. e. below some critical $\lambda_{c}$ ). However, the calculated critical values differed somewhat.

Rushbrooke, Stell, and Høye [158] present in 1973 the coexistence curve based on the MSA and a perturbation expansion ansatz by Stell, Rasaiah, and Narang 159]. An early Monte-Carlo calculation is undertaken by Ng, Valleau, and Torrie in 1979 [160] using only $N=32$ particles. Woodward and Nordholm 101] use three similar expressions for the free energy based on their effective potential (4.3) to calculate the coexistence curve. Kalikmanov et al. [13, 105] use the same effective potential and replace it by a related Lennard--Jones potential to find the critical point. Joslin and Goldman 25] finally calculate in 1993 the critical point by using second and third virial coefficients obtained from numerically calculated cluster integrals. The approximate results for the critical point $\left(\phi_{c}, \lambda_{c}\right)$ are summarized in the following table:

\begin{tabular}{r|c|c|c|c|c|c|c|c|} 
Work & {$[158](\mathrm{MSA})$} & {$[158](\mathrm{SRN})$} & {$[160]$} & {$[101] \mathrm{a}$} & {$[101] \mathrm{b}$} & {$[101] \mathrm{c}$} & {$[13]$} & {$[25]$} \\
\hline$\phi_{c}$ & 0.056 & 0.083 & 0.15 & 0.17 & 0.14 & 0.13 & 0.16 & 0.028 \\
$\lambda_{c}$ & 4.4 & 3.6 & 4.0 & 2.3 & 2.9 & 2.9 & 1.5 & 3.8
\end{tabular}

Despite the different predictions for the critical point there was a general belief at that time in the existence of a first-order dilute/dense phase transition. This changed when a couple of new Monte-Carlo simulations failed to detect such a transition. 
Caillol 161] performs a Monte-Carlo simulation with up to 512 particles and does not find a phase transition for two coupling constants $\lambda=4.5$ and $\lambda=5.55$ and volume fractions $0.08 \frac{\pi}{6}<\phi<0.38 \frac{\pi}{6}$. Furthermore, Monte-Carlo calculations by Weis and Levesque [137, 162] done for systems of similar size do not show a dilute/dense transition at low densities, $0.01<\phi<0.16$, even for couplings as strong as $\lambda=12.25$. Instead association of particles into chains is observed.

Van Leeuwen and Smit [163] consider a dipolar fluid with a modified Lennard-Jones short-range potential of the form

$$
v_{i j}^{S R}=4 v_{0}\left[\left(\frac{D}{r_{i j}}\right)^{12}-c\left(\frac{D}{r_{i j}}\right)^{6}\right] .
$$

The introduction of the parameter $c$ allows a smooth transition from dipolar soft spheres $(c=0)$ to Stockmayer particles $(c=1)$ [164]. For one fixed $v_{0}$ the authors do not find a dense-dilute phase transition if $c<0.3$ and conclude that the dipolar soft and hard sphere fluid will not exhibit such a transition. They observe chain formation instead.

Levesque and Weis 165] investigate the system of DHS with a dipolar coupling $\lambda=12.25$ in an even larger range of volume fractions, $0.005 \frac{\pi}{6}<\phi<0.8 \frac{\pi}{6}$, and in addition the range $4<\lambda<12.25$ for $\phi=0.01 \frac{\pi}{6}$ and $\phi=0.3 \frac{\pi}{6}$. For $\lambda=12.25$ they find long chains when $\phi<0.2 \frac{\pi}{6}$. For higher volume fractions the situation is less clear. Shorter chains can still be found applying an energetical criterion, but these chains are not directly visible in the configuration snapshots. At $\phi=0.6 \frac{\pi}{6}$ and higher they find a ferroelectric state (see below). They observe a continuous association of monomers into chains when increasing $\lambda$ for fixed volume fraction.

In 1994 and 1995 Stevens and Grest [157, 166] perform Monte-Carlo calculations for dipolar soft spheres both with and without applied magnetic field. They do not find a phase transition for the zero field case. Instead they observe also in this system chain formation similar to that of DHS. However, still in 1995 the same authors [152] report a dilute/dense phase transition for Monte-Carlo simulated Stockmayer fluids where the critical temperature measured in $1 / v_{0}$ and the critical density depend linearly on $\lambda / v_{0}$. The critical parameters agree well with those found by Hendriks, van Leeuwen et al. 154, 155, 156] who considered a smaller range of $\lambda / v_{0}$ already between 1989 and 1994 .

The association of ferrofluid particles into chains for $\lambda \gg 1$ and low densities is a natural consequence of the highly directional dipolar potential [167, 168]. The magnetic moments 
should be oriented in these chains in the energetically most favorable head-to-tail configuration with energy $-2 \lambda k_{\mathrm{B}} T$ for a pair of them. The numerical observation of chain formation instead of a homogeneous dilute/dense transition leads to a number of papers considering simple thermodynamic models of chains of dipolar hard spheres of similar particles. Small-angle neutron scattering experiments [169] have provided experimental support for the existence of such chain structures. Recently, a direct visual confirmation of the existence of chain structures in cryo-TEM images was achieved by Butter et al. 170].

Sear 171] argues that the chains should interact with each other only weakly and so he considers a model of only nearest-neighbor interacting particles, forming ideal chains. He compares with results of the simulations of [165] and 161]. Van Roij 172] investigates the competition between condensation due to isotropic interactions and chain formation due to dipolar forces.

The most detailed model was proposed by Osipov, Teixeira, and Telo da Gama [173, 174 who also take into account chain-chain interaction. They compare with results from [161] and [162]. These authors find semi-quantitative agreement concerning the critical points with the results of [163] for Stockmayer-like potentials. They predict a dilute/dense phase transition to appear also for particles with weak isotropic attraction (but not for DHS) where in both phases chaining should be observed. However, they also note that finite-size effects may make it difficult to observe this kind of transition in simulations. In 1999 Tavares, Weis, and Telo da Gama [175] improve the theory and perform own Monte-Carlo calculations at low densities $\phi=0.05 \frac{\pi}{6}$ and $5 \lesssim \lambda \lesssim 7.6$. Also in 1999 Levin [176] uses the Debye-HückelBjerrum theory to show that the clustering of particles into chains in the system of DHS should always reduce the density of free particles below the necessary density for a phase transition.

Klapp and Forstmann [177, 178] investigate Stockmayer and DHS fluids using the RHNC. There exists a parameter region of low temperatures where the RHNC fails to provide solutions. The boundary of this region is believed to be connected with phase transitions. At small densities the authors find growing fluctuations near this boundary that seem to represent the appearance of chains. There are analogous hints for a dilute/dense phase transition in the case of Stockmayer particles but not for DHS.

By the end of the 90s there thus was a kind of consensus reached that DHS do not show a dilute/dense phase transition because of the competing process of chaining. But then 
Shelley et al. 179] perform in 1999 Monte-Carlo simulations for dipolar hard dumbbells and spherocylinders. They find a dense/dilute phase coexistence even for almost spherical particles and observe, however, increasing sampling problems. They suggest that these sampling problems may have prevented the discovery of such a coexistence for DHS. Camp, Shelley, and Patey [180] therefore reinvestigate in 2000 the system of DHS with MonteCarlo simulations. For $\lambda=7.56$ they find indeed evidence for one or even two isotropic dense/dilute phase transitions. In the same year Camp and Patey [181] study the evolution of a system with $0.001 \frac{\pi}{6} \leq \phi \leq 0.6 \frac{\pi}{6}$. The simulation shows ring structures for the smallest $\phi$, chain structures for intermediate densities $\left(0.06 \frac{\pi}{6}<\phi<0.35 \frac{\pi}{6}\right)$, where the phase transitions reported in [180] take place, and a structureless dense liquid for still larger $\phi$.

Pshenichnikov and Mekhonoshin [182, 183] also perform Monte-Carlo simulations of DHS using a finite system. They find an ordinary dilute/dense transition with a critical point already at $\lambda_{c} \approx 3$ and $\phi_{c}=0.034$. This result is at odds with previous ones possibly because the authors of [182, 183] use instead of the generally preferred Ewald summation technique finite cylindrical and spherical systems with 1000 particles. They remark that periodic boundary conditions may cause errors in the case of long-range forces. However, a finite system with $N=2046$ particles was previously used by Levesque and Weis [165] for a volume fraction near $\phi_{c}$ and much higher $\lambda$ without finding a phase separation.

Morozov and Shliomis [184] study the intrachain correlations of chains of DHS for large $\lambda$ by performing a $1 / \lambda$-expansion of the needed statistical integrals. They find a persistence length of $\lambda / 2$ for $\lambda \rightarrow \infty$. Considering nonideal chains they predict a transition from chain to globule structures at $\lambda=3.2$

As discussed above, chaining in monodisperse systems has a strong influence on the dilute/dense phase transition. When comparing with experiments done on real ferrofluids it should therefore also be noted that polydispersity affects the lengths of chains as theoretically predicted by Kantorovich and Ivanov [185] and demonstrated in simulations by Wang and Holm [128].

The phase behavior of DHS and similar systems is further complicated when taking into account a nonvanishing magnetic field. Sano and Doi [100] investigate the dilute-dense phase transition in the presence of a magnetic field in a randomly filled lattice of dipolar particles. In zero field they find a rather small critical $\lambda$ and a rather large critical concentration in this system. In general a magnetic field makes a phase transition more likely and increases 
the density differences of coexisting phases. But there is a $\lambda$-interval near the zero-field $\lambda_{c}$ where a phase transition occurs for small and large fields but not for intermediate fields.

Stevens and Grest [152, 157, 166] who failed to find a dilute-dense transition for dipolar soft spheres in zero field demonstrate the existence of such a transition in Monte-Carlo simulations with applied fields. However, the two phases cannot be described as simple homogeneous gas and liquid phases since long chains are present in both of them. For both dipolar soft spheres and Stockmayer particles they show that $\lambda_{c}$ is getting smaller in applied fields whereas the value $\phi_{c} \approx 0.017$ remains mainly unchanged. In [173] however it is argued that this observed phase transition for soft spheres may be just an artifact of the insufficient system size.

Kusalik [186] does not find a phase separation in the system of dipolar soft spheres at high fields. However, his value of $\lambda \approx 3$ is smaller than those considered by Stevens and Grest who show that $\lambda_{c}>5$ even for infinite fields. The field dependence of $\lambda_{c}$ and $\phi_{c}$ in the case of Stockmayer particles is also studied with Monte-Carlo techniques by Boda et al. 187]. The authors find very similar results to those of Stevens and Grest. Szalai, Chan, and Tang [188] find also qualitatively the same behavior when they study dipolar Yukawa particles using the MSA.

Klapp and Forstmann [189] investigate perfectly aligned DHS, i. e., the infinite-field limit within the framework of the RHNC. They also do not find a dilute/dense phase transition up to $\lambda \approx 5$.

Zubarev and Iskakova investigate an interacting variant [190, 191] of their chain model that has already been used to calculate the equilibrium magnetization in [118, 119, 127] and show that in this model chain formation prevents a dilute/dense transition in the infinite field limit.

Morozov and Shliomis [184] find that nonideal chains gain stability against the transition to a globular cluster in applied fields.

\section{B. Ferromagnetic phases}

Here we review papers that have addressed the question whether the classical dipolar interaction between the nanoscale particles in a ferrofluid suffices to induce long-range orientational order of the magnetic dipoles in a liquid state without positional order, i.e., with 
homogeneously distributed particle positions.

Already the oldest model for the equilibrium magnetization of dipolar interacting particles, namely the Weiss model allows ferromagnetic solutions, i. e., a spontaneously generated equilibrium magnetization $M \neq 0$ for $H=0$ : Eq. (2.8) reads in a needle-shaped probe geometry for which $H=H_{e}$

$$
M=M_{\text {sat }} \mathcal{L}\left(\frac{m M}{3 k_{\mathrm{B}} T}\right)
$$

when $H=H_{e}=0$. This equation has solutions with $M \neq 0$ when $M_{\text {sat }} m / 3 k_{\mathrm{B}} T=\chi_{L}=8 \phi \lambda$ exceeds 3. Approaching this critical value, $\chi_{L}=3$, of the Langevin susceptibility the initial susceptibility (2.9) of the Weiss model undergoes a divergence. While the existence of a ferromagnetic state for such a rather large range, $\phi \lambda>3 / 8$, of parameters can be ruled out experimentally there are experimental hints for a ferromagnetic phase in the paper by Mamiya, Nakatani, and Furubayashi from 2000 [19] who find growing ferromagnetic fluctuations in their iron-nitride ferrofluid at low temperatures for $\phi \approx 0.15$.

It should be noted that the above mentioned divergence of $\chi$ disappears when the next diagonal term in the $(\phi, \lambda)$-expansion, i.e., the $\phi^{2} \lambda^{2}$-term is included in the expression for the equilibrium magnetization [64]. In this extended Weiss model one has

$$
M=M_{\text {sat }}\left[\frac{1}{3} \alpha_{\mathrm{eff}}-\frac{20}{9} \phi^{2} \lambda^{2} \alpha_{\mathrm{eff}}-\ldots \alpha_{\mathrm{eff}}^{3}+O\left(\alpha_{\mathrm{eff}}^{5}\right)\right],
$$

where $\alpha_{\text {eff }}=\frac{m}{k_{\mathrm{B}} T}\left(H+\frac{M}{3}\right)$ is the Weiss mean field. In this case the additional $\phi^{2} \lambda^{2}$-term prevents the existence of ferromagnetic solutions for $H=0$.

Zhang and Widom [192] extend the Weiss mean field theory describing the local field as a stochastic variable and find that ferromagnetic phases do not exist for any value of $\lambda$ if the volume fraction $\phi$ is smaller than some critical value 0.295.

At the end of the eighties theoretical investigations based on model Hamiltonians [193] or model distribution functions [194] lead to the prediction of ferromagnetic states only for particles with sufficient prolateness or oblateness. Nematic ferro- or antiferromagnetic phases were investigated with different methods in systems of dipolar ellipsoids and spherocylinders for example in [132, 195, 196, 197].

But in 1992 MD calculations by Wei and Patey [198, 199] show the existence of ferromagnetic states in the system of dipolar soft spheres for $v_{0}=1 / 1.35$ and $\lambda / v_{0}=9$ for volume fractions above $\phi \approx 0.65 \frac{\pi}{6}$. Since both, the soft and the hard sphere potential, are not only spherically symmetric but also purely repulsive both should behave very 
similar. Weis, Levesque, and Zarragoicoechea 200] find indeed in 1992 in Monte-Carlo simulations ferromagnetic fluid and solid phases in the system of DHS in their investigated range $0.8 \frac{\pi}{6}<\phi<1.2 \frac{\pi}{6}$ and $\lambda=6.25$. More results for this range are reported 1993 in [137]. Weis and Levesque [162] find ferromagnetic states also at lower volume fractions $\phi \approx 0.3 \frac{\pi}{6}$.

Wei, Patey, and Perera [201] use density-functional methods to investigate the transition to ferroelectric phases in systems of dipolar hard and soft spheres reproducing the numerical results qualitatively but not quantitatively. More systematic investigations of the phase diagrams of Stockmayer particles are undertaken by Groh and Dietrich [202, 203, 204] also using density functional methods. The critical temperatures are high. For $v_{0} \approx 0.3$ for example they find a transition between a dilute, isotropic fluid and a dense, ferroelectric fluid already for $\lambda / v_{0}=4$ or $\lambda \approx 1.3$.

Zhang and Widom [205] investigate the phase diagram starting with the expression for the free energy of a van-der-Waals fluid with two additional terms modeling the magnetic properties. The authors find qualitatively similar phase diagrams: For high temperatures there is a continuous transition from a dilute isotropic to a dense ferromagnetic liquid that becomes first order at smaller temperatures. If the short-range interaction energy is small enough compared to the dipolar interaction energy then there exists a temperature interval where the isotropic/ferromagnetic phase transition is preceded at smaller densities by a first-order transition between two isotropic phases having different densities.

Levesque and Weis [165] find ferromagnetic states for $\lambda=12.25$ and $\phi>0.6 \frac{\pi}{6}$. They however now argue that the ferromagnetic phase reported in $162 \mid$ for smaller $\phi$ was probably just a slowly decaying nonequilibrium state. Stevens and Grest [152, 157] find spontaneously magnetized fluid phases of Stockmayer particles for $\lambda=4$ and $\phi>0.47$. For soft spheres they find such magnetic fluid phases for the investigated values $4<\lambda<9$ when $\phi$ is greater than a value that depends linearly on $\lambda$. They see hints for a hysteresis in the magnetic-isotropic transition for $\lambda=16$.

Groh and Dietrich [206] study the phase transition properties of nonspherical dipolar hard particles using a density functional ansatz. In the special case of DHS they find no dilute/dense transition between isotropic phases but an isotropic/ferromagnetic transition that becomes noncontinuous at $\lambda>1.33$ and $\phi \approx 0.2$. The authors admit that the stability of the ferromagnetic phase is overestimated with respect to the simulations. That density functional methods are probably not useful for quantitative investigations of the phase be- 
havior is also pointed out by Osipov, Teixeira, and Telo da Gama [207] and in a different context by Ivanov [208] and Morozov [209].

Klapp and Forstmann [177, 178] find fluctuations at the high density boundary of the region of existence of isotropic RHNC solutions for the DHS and Stockmayer systems. They also find ferromagnetic solutions beyond that boundary.

Camp and Patey [181] report in 2000 that they found in their Monte-Carlo simulations ferromagnetic phases at $\lambda \approx 7.5$ for $\phi>0.5 \pi / 6$. A Monte-Carlo simulation of a DHS fluid with dipolar polydispersity, one with a bidisperse diameter distribution, and a binary mixture of dipolar and neutral hard spheres is undertaken by Cabral [210] for $\phi=0.8 \frac{\pi}{6}$ and $\lambda=6.25$. He shows that the polydispersity reduces the ferromagnetic order.

In 2003 Ivanov [208] investigates the BBGKY equation relating the one-particle distribution function $\rho(\mathbf{r}, \mathbf{m})$ to the pair correlation function $g(1,2)$. He employs for the latter the self-consistent expression used in density functional approaches [189, 201, 202, 204]. When expanding it up to second order in the dipolar interaction the so approximated BBGKY equation shows a bifurcation to a solution with spontaneously generated ferrimagnetic longrange order for $\chi_{L}>12(\sqrt{5}-2) \simeq 2.83$, i.e., slightly below the bifurcation threshold for ferromagnetism in the Weiss model. Ivanov [208] argues that this peculiar ferrimagnetic behavior is an artifact that is ultimately due to the mean-field character of the density functional ansatz for the pair correlation function.

Then in 2003 Morozov 209] investigates the bifurcation properties of the so-called LovettMou-Buff-Gubbins integral equation [112, 113] that relates the one-particle probability distribution function to the direct correlation function $c(1,2)$ and that is equivalent to the above mentioned first BBGKY equation. He establishes criteria for the bifurcation of solutions with long-range magnetic order in ellipsoidal and spherical sample shapes in vacuum and compares with mean-field predictions, density function theory, and MSA results. Using a generalized MSA type expression for $c(1,2)$ he finds that the strength of short-range correlations plays a decisive role for the appearance of spontaneous long-range magnetic order - the susceptibility diverges when the former exceed a critical strength. More qualitative arguments then show that the short-range correlations are most likely to be antiferromagnetic. He also shows that approximating $c(1,2)$ by the two lowest orders in its diagram expansion always gives rise to liquid ferromagnetic solutions.

At the end of this section on phase transitions in ferrofluids we should like to quote 
Teixeira, Tavares and Telo da Gama [211]. They have presented in 2000 a fairly extensive review on the effect of dipolar forces on the structure and thermodynamics of classical fluids that covers many aspects of the problems related to gas/liquid condensation phase transitions in various dipolar systems. Since their summary of the state of research on these problems still gives in our opinion an adequate picture of today's state of the art - also concerning the problem of liquid ferromagnetism — we give two quotes [211]: "Although widely studied in statistical mechanics, the phase diagrams of dipolar fluids in general, and of strongly dipolar fluids in particular, have remained largely uncharted" and "In conclusion, it is fair to say that a theory is not yet available that is capable of describing dipolar fluids over the whole range of densities and dipole strengths. Moreover, the mechanisms driving the phase transitions (as well as the location of the phase boundaries) remain unclear." 


\section{CONCLUSION}

Ferrofluids, i.e., suspensions of magnetic nano-particles in liquid carriers are not only of technological interest but they continue to be also the object of many basic research projects. Among those has the effect of dipolar interactions that are important in ferrofluids attracted considerable interest for two reasons: First, dipolar forces are long-range so that, e.g., the equilibrium magnetization (or polarization) is geometry dependent. Second, dipolar forces are attractive or repulsive depending on the relative orientation of the particles and their dipole moments with the orientational mean being zero. A realistic theoretical description of the colloidal suspension will also take into account other interactions between the particles and in addition the dispersion of particle diameters and magnetic moments in ferrofluids that is absent in molecular systems. The dipolar interaction affects naturally the equilibrium magnetization, but also the phase transition behavior. These two subjects were reviewed here.

The question of how dipolar interactions influence the initial susceptibility $\chi$ was studied for electrically polar systems long before the advent of ferrofluids. The oldest models, the Weiss and Onsager models, to capture this influence with mean-field approaches were proposed already in the first half of the 20th century. Only after 1970 other theoretical methods were used intensively to investigate $\chi$ by cluster expansions, integral equations, and Monte-Carlo and molecular dynamics simulations. Here mainly dipolar hard spheres and Stockmayer particles were investigated. While both represent only poorly polar molecules like water they are much better approximations to the nanoscale magnetic particles of ferrofluids. In fact the latter seem to be very good realizations of these two model systems. Many of the earlier analytical and semi-analytical theories that originally were conceived for molecular systems were also extended to account for polydispersity in ferrofluids.

When comparing cluster expansion methods and integral equation methods with numerical simulations it seems that the RHNC theory and the simpler perturbation theory by Tani et al. 26] agree best with the Monte-Carlo results for $\chi$, at least for relatively high densities. The second theory has also been applied to explain experimental data for a highly concentrated ferrofluid, as has a related cluster expansion result by Ivanov and Kuznetsova [124].

With the synthetization of ferrofluids, i.e., dipolar systems in which saturation could 
easily be reached not only $\chi$ but also the full magnetization curve $M(H)$ became of interest. Here, the main theoretical results date from 1985 onwards. The proposed theories were mostly extensions of those already used to describe $\chi$. For example, the one by Ivanov and Kuznetsova [124] compares well with experimental data. However, recent numerical simulations by Wang et al. [126, 128] for $\chi$ and $M(H)$ are not well described by this theory when the volume fraction of the magnetic particles is small and the dipolar coupling is strong: they are better explained by a model of small non-interacting chains of different lengths.

An ever increasing analytical, numerical, and experimental research activity has been devoted to phase transition phenomena in dipolar systems, namely, $(i)$ the separation into dilute and dense phases and (ii) the appearance of spontaneous (ferromagnetic) long-range order in zero magnetic field. Both phenomena refer in the case of ferrofluids solely to the subsystem of the magnetic particles without positional long-range order.

Dilute/dense transitions occur in Lennard--Jones systems and, more generally, whenever the attractive part of the interaction is sufficiently strong. In fact simulations have shown that switching on an additional dipolar interaction in such systems favors the transition and increases the critical temperature. However, the question whether such a phase transition occurs also for purely dipolar hard spheres without any isotropic attraction is more difficult to answer. The fact that such phase separation has been observed experimentally in real ferrofluids does not help much to solve the above theoretical problem when (strong) isotropic interactions cannot be excluded to be present in the ferrofluid.

In the 1970s and 1980s a variety of semi-analytical theories was applied to investigate the dilute/dense phase transition behavior of dipolar hard spheres. They all found a usual phase diagram with a roughly parabolic coexistence curve. However, there was no agreement about the location of the critical point. But Monte-Carlo simulations in the 90s did not find such phase transitions for dipolar hard spheres. For strong enough dipolar couplings or, equivalently, low enough temperatures a formation of head-to-tail dipolar chains was observed instead. They are caused by the highly directional character of the dipolar pair potential. Thus, new models were developed to explain the observed behavior by means of polymer theory. According to these models the assembly of chains prevent a usual dilute/dense transition or mask it and make its observation in simulations difficult due to finite-size effects. 
Another turning point was reached in 2000 when Camp et al. [180] found evidence for one or even two phase transitions between chain-dominated states in Monte-Carlo simulations. Pshenichnikov and Mekhonoshin [182, 183] even observed the common dilute/dense transition already at quite low dipolar couplings where chain formation is not very effective. They explain the differences to other studies with their choice of a finite system instead of periodic boundary conditions. The differences between both simulation methods remain to be investigated further.

The phase transition behavior is complicated further by the presence of a magnetic field. In the case of Stockmayer particles the field supports the phase separation, i. e., it enlarges the coexistence region. The question whether a field can trigger such a phase separation also for dipolar hard spheres is not yet settled.

In comparison to the question of a dilute/dense transition the research results concerning the possibility of having a phase with spontaneous (ferromagnetic) long-range order in zero magnetic field seem to be more coherent - albeit only on first sight: Experimental hints for such a phase were found by Mamiya et al. 19] and the simulations performed since the 1990s generally agree that there exist ferromagnetic phases for dipolar hard spheres and similar systems in regions of high densities and strong couplings. This behavior was also found in density functional theories although they seem to be less suited to explain the phase behavior and the results differ quantitatively. The conditions for the appearance of spontaneous longrange magnetic order were recently investigated by Morozov [209] and Ivanov [208] using integral equation methods. They conclude that mean-field type approximations that are also the core of density functional approaches tend to generate artificially long-range magnetic order.

So, all in all we think that a lot of questions/problems related to the equilibrium behavior of ferrofluids and dipolarly interacting particles remain to be addressed.

\section{Acknowledgments}

We thank K. Morozov for helpful discussions and for critically reading the manuscript. This work was supported by the DFG (SFB 277) and by INTAS (Ref. Nr. 03-51-6064). 
[1] R. E. Rosensweig: Ferrohydrodynamics, Cambridge University Press, New York (1985).

[2] A. Aharoni: Introduction to the theory of ferromagnetism, 2nd edition, Oxford University Press, Oxford (2000).

[3] N. Spaldin: Magnetic materials, fundamentals and device applications, Cambridge University Press, Cambridge (2003).

[4] J. L. Dormann, D. Fiorani: Magnetic properties of fine particles, North Holland, Amsterdam (1992).

[5] J. L. Garcia-Palacios: On the statics and dynamics of magnetoanisotropic nanoparticles, in Advances in Chemical Physics, Vol. 112, edited by I. Prigogine and S. A. Rice J. Wiley, New York (2000).

[6] S. Odenbach, S. Thurm, K. Melzner: Introduction to the magnetic fluids bibliography, J. Magn. Magn. Mater. 252, 421 (2002).

[7] Magnetic Fluids Bibliography (1998-2001), J. Magn. Magn. Mater. 252, 422 (2002).

[8] B. M. Berkovsky, V. F. Medvedev, M. S. Krakov: Magnetic fluids, engineering applications Oxford University Press, Oxford (1993).

[9] B. M. Berkovsky, V. G. Bashtovoy: Magnetic fluids and applications handbook Begell House Inc., New York (1996).

[10] E. Blums, A. Cebers, M. M. Maiorov: Magnetic Fluids Walter de Gruyter, Berlin (1997).

[11] S. Odenbach: Magnetoviscous effects in ferrofluids, Lecture Notes in Physics, m 71, Springer, Berlin (2002).

[12] S. Odenbach: Ferrofluids - Magnetically controllable fluids and their applications, Lecture Notes in Physics, 594, Springer, Berlin (2002).

[13] V. I. Kalikmanov: Statistical thermodynamics of ferrofluids, Physica A 183, 25 (1992).

[14] P. Debye: Einige Resultate einer kinetischen Theorie der Isolatoren, Physik. Zeits. 13, 97 (1912).

[15] L. Onsager: Electric moments of molecules in liquids, J. Amer. Chem. Soc. 58, 1486 (1936).

[16] S. Khan, T. L. Morton, D. Ronis: Static correlations in macro-ionic suspensions: Analytic and numerical results in a hypernetted-chain-mean-spherical approximation, Phys. Rev. A 35, 4295 (1987). 
[17] T. Sato, T. Iijima, M. Seki, N. Inagaka: Magnetic properties of ultrafine ferrite particles, J. Magn. Magn. Mater. 65, 252 (1987).

[18] G. A. van Ewijk, G. J. Vroege, A. P. Philipse: Susceptibility measurements on a fractionated aggregate-free ferrofluid, J. Phys.: Condens. Matter 14, 4915 (2002).

[19] H. Mamiya, I. Nakatani, T. Furubayashi: Phase transition of iron-nitride magnetic fluids Phys. Rev. Lett. 84, 6106 (2000).

[20] D. W. Jepsen: Calculation of the dielectric constant of a fluid by cluster expansion methods, J. Chem. Phys. 44, 774 (1966).

[21] G. S. Rushbrooke: On the dielectric constant of dipolar hard spheres, Molec. Phys. 37, 761 (1979).

[22] C. G. Joslin: The third dielectric and pressure virial coefficient of dipolar hard spheres fluids, Molec. Phys. 42, 1507 (1981).

[23] A. D. Buckingham, C. G. Joslin: The second dielectric virial coefficient of dipolar hard-sphere fluids, Molec. Phys. 40, 1513 (1980).

[24] G. S. Rushbrooke, B. G. Shrubsall: On the third dielectric virial coefficient for dipolar hard spheres, Molec. Phys. 55, 599 (1985).

[25] C. Joslin, S. Goldman: The third dielectric and pressure virial coefficient of dipolar hard spheres fluids II: Numerical results, Molec. Phys. 79, 499 (1993).

[26] A. Tani, D. Henderson, J. A. Barker, C. E. Hecht: Application of perturbation theory to the calculation of the dielectric constant of a dipolar hard spheres fluid, Molec. Phys. 48, 863 (1983).

[27] S. Goldman: Determination of static dielectric constant-temperature-density surfaces of a Stockmayer fluid by perturbation theory, Molec. Phys. 71, 491 (1990).

[28] J. P. Hansen, I. R. McDonald: Theory of simple liquids, 2nd edition, Academic Press, New York (1990).

[29] M. S. Wertheim: Exact solution of the mean spherical model for fluids of hard spheres with permanent electric dipole moments, J. Chem. Phys. 55, 4291 (1971).

[30] G. Nienhuis, J. M. Deutch: Comparison of two theories for the two-particle distribution function of polar fluids, J. Chem. Phys. 56, 5511 (1972).

[31] J. L. Lebowitz, J. K. Percus: Mean spherical model for lattice gases with extended hard cores and continuum fluids, Phys. Rev. 144, 251 (1966). 
[32] L. Verlet, J. J. Weis: Perturbation theories for polar fluids, Molec. Phys. 28, 665 (1974).

[33] G. Stell, J. J. Weis: Pair correlation in simple polar liquids, Phys. Rev. A 16, 757 (1977).

[34] G. N. Patey: An integral equation theory for the dense dipolar hard-sphere fluid, Molec. Phys. 34, 427 (1977).

[35] M. S. Wertheim: Theory of polar fluids I, Molec. Phys. 26, 1425 (1973).

[36] G. N. Patey, D. Levesque, J. J. Weis: Integral equation approximation for dipolar fluids, Molec. Phys. 38, 219 (1979).

[37] Yu. V. Agrafonov, G. A. Martinov, G. N. Sarkisov: Solution of the hypernetted-chain equation for dipolar liquids by perturbation theory techniques, Molec. Phys. 39, 963 (1980).

[38] D. Y. C. Chan, G. R. Walker: A non-linear truncation scheme for the Ornstein-Zernike equation for dipolar fluids, Molec. Phys. 47, 881 (1982).

[39] P. H. Fries, G. N. Patey: The solution of the hypernetted-chain approximation for fluids of nonspherical particles. A general method with application to dipolar hard spheres, J. Chem. Phys. 82, 429 (1985).

[40] L. Y. Lee, P. H. Fries, G. N. Patey: The solution of the reference hypernetted chain approximation for Stockmayer fluids, Molec. Phys. 55, 751 (1985).

[41] P. H. Fries, G. N. Patey: The solution of the Percus-Yevick approximation for fluids with angle-dependent pair interactions. A general method with results for dipolar hard spheres, J. Chem. Phys. 85, 7307 (1986).

[42] F. Lado: Structure and thermodynamics of the dipolar hard sphere fluid from the referencehypernetted chain equation with minimized free energy, J. Chem. Phys. 85, 2916 (1986).

[43] E. Lomba, C. Martin, M. Lombardero: The dipolar hard sphere fluid: Hypernetted chain approximation revisited, Molec. Phys. 77, 1005 (1992).

[44] D. J. Adams: Computer simulations of highly polar liquids: The hard sphere plus point dipole potential, Molec. Phys. 40, 1261 (1980).

[45] D. J. Adams, E. M. Adams: Static dielectric properties of the Stockmayer fluid from computer simulations, Molec. Phys. 42, 907 (1981).

[46] D. J. Adams, I. R. McDonald: Thermodynamic and dielectric properties of polar lattices, Molec. Phys. 32, 931 (1976).

[47] J. A. Barker, R. O. Watts: Monte Carlo studies of the dielectric properties of water-like models, Molec. Phys. 26, 789 (1973). 
[48] G. N. Patey, J. P. Valleau: The free energy of spheres with dipoles: Monte Carlo with multistage sampling, Chem. Phys. Lett. 21, 297 (1973).

[49] G. N. Patey, J. P. Valleau: Dipolar hard spheres a Monte Carlo study, J. Chem. Phys. 61, 534 (1974).

[50] D. Levesque, G. N. Patey, J. J. Weis: A Monte Carlo study of dipolar hard spheres: The pair distribution function and the dielectric constant, Molec. Phys. 34, 1077 (1977).

[51] G. N. Patey, D. Levesque, J. J. Weis: On the theory and computer simulations of dipolar fluids, Molec. Phys. 45, 733 (1982).

[52] F. Lado: Perturbation approach to the computer simulations of dipolar fluids, J. Chem. Phys. 85, 2913 (1986).

[53] E. L. Pollock, B. J. Alder: Static dielectric properties of Stockmayer fluids, Physica A 102, 1 (1980).

[54] D. Levesque, J. J. Weis: On the calculation of dielectric properties from computer simulations, Physica A 125, 270 (1984).

[55] D. J. Evans, G. P. Morris: Computation of dielectric constants for condensed phases, Phys. Rev. A 33, 1408 (1986).

[56] M. Neumann, O. Steinhauser, G. Pawley: Consistent calculation of the static and frequencydependent dielectric constant in computer simulations, Molec. Phys. 52, 97 (1984).

[57] C. Hesse-Bezot, G. Bossis, C. Brot: New molecular dynamics simulation of a 3D fluid of Stockmayer and modified Stockmayer particles, J. Chem. Phys. 80, 3399 (1984).

[58] M. Neumann: Dipole moment fluctuation formulas in computer simulations of polar systems, Molec. Phys. 50, 841 (1983).

[59] M. Neumann, O. Steinhauser: The influence of boundary conditions used in machine simulations on the structure of polar systems, Molec. Phys. 39, 437 (1980).

[60] M. Neumann, O. Steinhauser: On the calculation of the dielectric constant using the EwaldKornfeld tensor, Chem. Phys. Lett. 95, 417 (1983).

[61] C. G. Gray, Y. S. Sainger, C. G. Joslin, P. T. Cummings, S. Goldman: Computer simulations of dipolar fluids. Dependence of the dielectric constant on system size, J. Chem. Phys. 85, 1502 (1986).

[62] Z. Wang, C. Holm: Estimate of the cutoff errors in the Ewald summation for dipolar systems, J. Chem. Phys. 115, 6351 (2001). 
[63] Z. Wang, C. Holm, H. W. Müller: Boundary condition effects in the simulation study of equilibrium properties of magnetic dipolar fluids, J. Chem. Phys. 119, 379 (2003).

[64] B. Huke, M. Lücke: Magnetization of ferrofluids with dipolar interactions: A Born-Mayer expansion, Phys. Rev. E 62, 6875 (2000).

[65] G. A. Chapela, E. Martina: The dielectric constant of dipolar sticky hard spheres, Molec. Phys. 55, 97 (1985).

[66] C. G. Joslin, C. G. Gray: The second dielectric virial coefficient of a dipolar sticky hard sphere fluid, Molec. Phys. 57, 1197 (1986).

[67] P. G. Kusalik: A comparison between computer simulation and theoretical results for fluids of dipolar soft spheres, Molec. Phys. 67, 67 (1989).

[68] P. G. Kusalik: Computer simulation results for the dielectric properties of a highly polar fluid, J. Chem. Phys. 93, 3520 (1990).

[69] P. G. Kusalik: On the computer simulation of highly polar fluids using large systems, Molec. Phys. 73, 1349 (1991).

[70] D. Henderson, D. Boda, I. Szalai, K.-Y. Chan: The mean spherical approximation for a dipolar Yukawa fluid, J. Chem. Phys. 110, 7348 (1999).

[71] I. Szalai, D. Henderson, D. Boda, K.-Y. Chan: Thermodynamics and structural properties of the dipolar Yukawa fluid, J. Chem. Phys. 111, 337 (1999).

[72] S. A. Adelman, J. M. Deutch: Exact solution of the mean spherical model for simple polar mixtures, J. Chem. Phys. 59, 3971 (1973).

[73] D. Isbister, R. J. Bearman: Solution of the mean spherical model for dipolar mixtures, Molec. Phys. 28, 1297 (1974).

[74] B. Freasier, N. Hamer, D. Isbister: Dielectric properties of a mixture of dipolar hard spheres, Molec. Phys. 38, 1661 (1979).

[75] J. D. Ramshaw, N. D. Hamer: Existence of the dielectric constant in dipolar fluid mixtures, J. Chem. Phys. 75, 3511 (1981).

[76] P. T. Cummings, L. Blum: Dielectric constant of dipolar hard sphere mixtures, J. Chem. Phys. 85, 6658 (1986).

[77] P. H. Lee, B. M. Ladanyi: Structural and dielectric properties of dipolar hard sphere mixtures, J. Chem. Phys. 87, 4093 (1987).

[78] P. H. Lee, B. M. Ladanyi: Structural and dielectric properties of dipolar hard sphere mixtures: 
Reference hypernetted chain an perturbation theory results, J. Chem. Phys. 91, 7063 (1989).

[79] K. O'Grady, A. Bradbury, S. W. Charles, S. Menear, J. Popplewell, R. W. Chantrell: CurieWeiss behavior in ferrofluids, J. Magn. Magn. Mater. 31-34, 958 (1983).

[80] S. Menear, A. Bradbury, R. W. Chantrell: Ordering temperatures in ferrofluids, J. Magn. Magn. Mater. 39, 17 (1983).

[81] S. Menear, A. Bradbury, R. W. Chantrell: A model of the properties of colloidal dispersions weakly interacting fine ferromagnetic particles, J. Magn. Magn. Mater. 43, 166 (1984).

[82] A. Bradbury, S. Menear, R. W. Chantrell: Concentration dependence of the ordering temperature in magnetic liquids, J. Appl. Phys. 57, 4265 (1985).

[83] A. Bradbury, S. Menear, R. W. Chantrell: A Monte Carlo calculation of the magnetic properties of a ferrofluid containing interacting polydispersed particles, J. Magn. Magn. Mater. 5457, 745 (1986).

[84] A. Bradbury, G. A. R. Martin, R. W. Chantrell: Zero field particle correlations in a ferrofluid using Monte Carlo simulations and the hypernetted chain approximation, J. Magn. Magn. Mater. 65, 6 (1987).

[85] G. A. R. Martin, A. Bradbury, R. W. Chantrell: A comparison between a threedimensional Monte Carlo model and a hypernetted chain approximation of a ferrofluid, J. Magn. Magn. Mater. 65, 177 (1987).

[86] J. Popplewell, B. Abu Aisheh, N. Y. Ayoub: The effect of particle interactions on CurieWeiss behavior in ferrofluids, J. Appl. Phys. 64, 5852 (1988).

[87] M. Holmes, K. O'Grady, J. Popplewell: A study of Curie-Weiss behavior in ferrofluids, J. Magn. Magn. Mater. 47, 47 (1990).

[88] H. D. Williams, K. O'Grady, S. W. Charles, K. J. Davies: Curie-Weiss behavior in ferrofluids: the effects of particle size and distribution, J. Magn. Magn. Mater. 122, 134 (1993).

[89] K. I. Morozov, A. F. Pshenichnikov, Yu. I. Raikher, M. I. Shliomis: Magnetic properties of ferrocolloids: The effect of interparticle interactions, J. Magn. Magn. Mater. 65, 269 (1987).

[90] A. F. Pshenichnikov, A. V. Lebedev, K. I. Morozov: Effect of interaction between particles on the magnetic properties of magnetic liquids, Magnetohydrodynamics 23, 31 (1987).

[91] M. I. Shliomis, A. F. Pshenichnikov, K. I. Morozov, I. Yu. Shurubor: Magnetic properties of ferrocolloids, J. Magn. Magn. Mater. 85, 40 (1990).

[92] V. I. Kalikmanov: Algebraic perturbation theory for polar fluids: A model for the dielectric 
constant, Phys. Rev. E 59, 4085 (1999).

[93] A. F. Pshenichnikov: Equilibrium magnetization of concentrated ferrocolloids, J. Magn. Magn. Mater. 145, 319 (1995).

[94] Yu. A. Buyevich, A. O. Ivanov: Equilibrium properties of ferrocolloids, Physica A 190, 276 (1992).

[95] I. Szalai, K.-Y. Chan, D. Henderson: Comment on "Algebraic perturbation theory for polar fluids: A model for the dielectric constant" , Phys. Rev. E 62, 8846 (2000); V. I. Kalikmanov: Reply to "Comment on 'Algebraic perturbation theory for polar fluids: A model for the dielectric constant'", Phys. Rev. E 62, 8851 (2000).

[96] A. F. Pshenichnikov, V. V. Mekhonoshin: Equilibrium magnetization and microstructure of the system of superparamagnetic interacting particles: Numerical simulation, J. Magn. Magn. Mater. 213, 357 (2000).

[97] A. Tari, R. W. Chantrell, S. W. Charles, J. Popplewell: The magnetic properties and stability of a ferrofluid containing $\mathrm{Fe}_{3} \mathrm{O}_{4}$ particles, Physica B 97, 57 (1979).

[98] J. S. Høye, G. Stell: Statistical mechanics of dielectric fluids in electric fields: A mean field treatment, J. Chem. Phys. 75, 3559 (1981).

[99] Yu. I. Dikanskii: Experimental investigation of effective magnetic fields in a magnetic fluid, Magnetohydrodynamics 18, 237 (1982).

[100] K. Sano, M. Doi: Theory of agglomeration of ferromagnetic particles in magnetic fluids, J. Phys. Soc. Jpn 52, 2810 (1983).

[101] C. E. Woodward, S. Nordholm: An effective pair potential for dipolar fluids: The coexistence curve, Molec. Phys. 52, 973 (1984).

[102] C. E. Woodward, S. Nordholm: Density functional theory of dipolar fluids: I. An effective pair potential for orientational correlations in an electric field, Molec. Phys. 59, 1177 (1986).

[103] C. E. Woodward, S. Nordholm: Nonlinear electric field effects on the thermodynamic properties of dipolar fluids, J. Phys. Chem. 92, 501 (1988).

[104] B. M. Berkovsky, V. I. Kalikmanov, V. S. Filinov: On a statistical theory of magnetic fluids, J. Phys. C 18, L945 (1985).

[105] B. M. Berkovsky, V. I. Kalikmanov, V. S. Filinov: On equilibrium properties and phase diagram of magnetic fluids, J. Magn. Magn. Mater. 65, 191 (1987).

[106] V. N. Bagaev, Yu. A. Buyevich, A. O. Ivanov: The theory of the magnetic properties of 
ferrocolloids, Magnetohydrodynamics 25, 52 (1989).

[107] A. O. Ivanov: The theory of the magnetostatic properties of polydisperse ferrofluids, Magnetohydrodynamics 25, 460 (1989).

[108] Yu. A. Buyevich, A. O. Ivanov, A. Yu. Zubarev: Statistical thermodynamics of ferrocolloids, J. Magn. Magn. Mater. 85, 33 (1990).

[109] A. O. Ivanov: Magnetostatic properties of moderately concentrated ferrocolloids, Magnetohydrodynamics 28, 353 (1992).

[110] A. V. Lebedev: Calculating the magnetization curves of concentrated magnetic fluids, Magnetohydrodynamics 25, 520 (1989).

[111] K. I. Morozov, A. V. Lebedev: The effect of magneto-dipole interactions on the magnetization curves of ferrofluids, J. Magn. Magn. Mater. 85, 51 (1990).

[112] R. Lovett, C. Y. Mou, F. P. Buff: The structure of the liquid-vapor interface, J. Chem. Phys. 65, 570 (1976).

[113] K. E. Gubbins: Structure of non-uniform molecular fluids: Integrodifferential equations for the density-orientation profile, Chem. Phys. Lett. 76, 329 (1980).

[114] S. Menear, A. Bradbury: The effects of weak dipolar interactions on the magnetic properties of systems of fine ferromagnetic particles, J. Magn. Magn. Mater. 51, 103 (1985).

[115] A. Bradbury, G. Martin: A theoretical study of the thermodynamic behavior of a ferrofluid, J. Phys. Chem. 97, 2830 (1993).

[116] N. Y. Ayoub, A. Shamoun, A. K. Abdallah, M. S. Dababneh, I. M. Odeh, N. M. Laham: The magnetic properties of a uniform-size fine-particle ferrofluid in the dimer model in three dimensions, J. Magn. Magn. Mater. 127, 75 (1993).

[117] In 115] the last term in Eq. (44) must $\operatorname{read} 32\left(\sinh \alpha / \alpha-2 \cosh \alpha / \alpha^{2}+2 \sinh \alpha / \alpha^{3}\right)^{2}$. In [116] the last term in Eq. (12) must read $3 i_{2}^{2}(\alpha) / \alpha^{2}$.

[118] A. Yu. Zubarev, L. Yu. Iskakova: Theory of physical properties of magnetic liquids with chain aggregates, JETP 80, 857 (1996).

[119] I. Abu-Aljarayesh, Sh. Migdadi: Numerical calculations of the entropy and magnetization of magnetic fluids with chain aggregates, J. Magn. Magn. Mater. 191, 174 (1999).

[120] A. F. Pshenichnikov, V. V. Mekhonoshin, A. V. Lebedev: Magneto-granulometric analysis of concentrated ferrocolloids, J. Magn. Magn. Mater. 161, 94 (1996).

[121] B. Huke, M. Lücke: Magnetization of concentrated polydisperse ferrofluids: Cluster expan- 
sion, Phys. Rev. E 67, 051403 (2003).

[122] S. H. L. Klapp, G. N. Patey: Integral equation theory for dipolar hard sphere fluids with fluctuating orientational order, J. Chem. Phys. 112, 3832 (2000).

[123] M. S. Wertheim: Correlations in the liquid-vapor interface, J. Chem. Phys. 65, 2377 (1976).

[124] A. O. Ivanov, O. B. Kuznetsova: Magnetic properties of dense ferrocolloids: Influence of interparticle correlations, Phys. Rev. E 64, 041405 (2001).

[125] A. O. Ivanov: private communication

[126] Z. Wang, C. Holm, H. W. Müller: Molecular dynamics study on the equilibrium magnetization properties and structure of ferrofluids, Phys. Rev. E 66, 021405 (2002).

[127] A. O. Ivanov, Z. Wang, C. Holm: Applying the chain formation model to magnetic properties of aggregated ferrofluids, Phys. Rev. E 69, 031206 (2004).

[128] Z. Wang, C. Holm: Structure and magnetic properties of polydisperse ferrofluids: A molecular dynamics study, Phys. Rev. E 68, 041401 (2003).

[129] T. Kristof, I. Szalai: Magnetic properties and structure of polydisperse ferrofluid models, Phys. Rev. E. 68, 041109 (2003).

[130] M. Raşa, B. Bica, A. Philipse, L. Vékás: Dilution series approach for investigation of microstructural properties and particle interactions in high-quality magnetic fluids, Eur. Phys. J. E 7, 209 (2002).

[131] C. G. Joslin: Second virial coefficients for a fluid of dipolar hard ellipsoids, Molec. Phys. 47, $771(1982)$.

[132] A. Perera, G. N. Patey: Fluids of dipolar hard ellipsoids: Structural properties and isotropicnematic phase transitions, J. Chem. Phys. 91, 3045 (1989).

[133] S. W. de Leeuw, N. Quirke: Dielectric behavior and multibody orientational correlations of dipolar molecular liquids, J. Chem. Phys. 81, 880 (1984).

[134] E. Lomba, M. Lombardero, J. L. F. Abascal: New aspects in the simulation and behavior of polar molecular fluids, Molec. Phys. 68, 1067 (1989).

[135] G. N. Patey, D. Levesque, J. J. Weis: Integral equation approximations for fluids of hard spheres with dipoles and quadrupoles, Molec. Phys. 38, 1635 (1979).

[136] S. L. Carnie, G. N. Patey: Fluids of polarizable hard spheres with dipoles and tetrahedral quadrupoles, Molec. Phys. 47, 1129 (1982).

[137] J. J. Weis, D. Levesque: Ferroelectric phases of dipolar hard spheres, Phys. Rev. E 48, 3728 
(1993).

[138] B. Groh, S. Dietrich: Density-functional theory for the freezing of Stockmayer fluids, Phys. Rev. E 54, 1687 (1996).

[139] B. Groh, S. Dietrich: Crystal structures and freezing of dipolar fluids, Phys. Rev. E 63, 021203 (2001).

[140] X. S. Chen, M. Kasch, F. Forstmann: Demixing phase transition in a mixture of hard-sphere dipoles and neutral hard spheres, Phys. Rev. Lett. 67, 2674 (1991).

[141] W. Luo, S. R. Nagel, T. F. Rosenbaum, R. R. Rosensweig: Dipole interactions with random anisotropy in a frozen ferrofluid, Phys. Rev. Lett. 67, 2721 (1991).

[142] B. Mertens, K. Levin, G. S. Grest: Magnetic properties of a frozen ferrofluid: Local-meanfield theory, Phys. Rev. B 49, 15374 (1994).

[143] G. Ayton, M. J. P. Gingras, G. N. Patey: Ferroelectric and dipolar glass phases of noncrystalline systems, Phys. Rev. E 56, 562 (1997).

[144] A. A. Minakov, I. A. Zaitsev, U. I. Lesnih: Critical behavior of magnetic fluids near superparamagnetic dipole-glass transitions, J. Magn. Magn. Mater. 135, 237 (1995).

[145] Yu. V. Burnishev, Yu. I. Rozenberg: Magnetization dynamics of ferrocolloids near the hardening interval, J. Magn. Magn. Mater. 85, 60 (1990).

[146] B. E. Vugmeister, M. D. Glinchuk: Dipole glass and ferroelectricity in random-site electric dipole systems, Rev. Mod. Phys. 62, 993-1026 (1990).

[147] L. N. Donselaar, P. M. Frederik, P. Bomans, P. A. Buining, B. M. Humbel, A. P. Philipse: Visualization of particle association in magnetic fluids in zero-field, J. Magn. Magn. Mater. 201, 58 (1999).

[148] J.-C. Bacri, R. Perzynski, D. Salin, V. Cabuil, R. Massart: Phase diagram of an ionic magnetic colloid: Experimental study of the effect of ionic strength, J. Colloid Interface Sci. 132, $43(1989)$.

[149] J.-C. Bacri, R. Perzynski, D. Salin, V. Cabuil, R. Massart: Ionic ferrofluids: A crossing of chemistry and physics, J. Magn. Magn. Mater. 85, 27 (1990).

[150] R. Massart, E. Dubois, D. Salin, V. Cabuil, E. Hasmonay: Preparation and properties of monodisperse magnetic fluids, J. Magn. Magn. Mater. 149, 1 (1995).

[151] F. Cousin, E. Dubois, V. Cabuil: Tuning the interaction of a magnetic colloidal suspension, Phys. Rev. E. 68, 021405 (2003). 
[152] M. J. Stevens, G. S. Grest: Structure of soft-sphere dipolar liquids, Phys. Rev. E 51, 5962 (1995).

[153] P. Frodl, S. Dietrich: Bulk and interfacial properties of polar and molecular fluids, Phys. Rev. A 45, 7330 (1992).

[154] B. Smit, C. P. Williams, E. M. Hendriks,S. W. de Leeuw: Vapor-liquid equilibria for Stockmayer fluids, Molec. Phys. 68, 765 (1989).

[155] M. E. van Leeuwen, B. Smit, E. M. Hendriks: Vapor-liquid equilibria for Stockmayer fluids: Computer simulations and perturbation theory, Molec. Phys. 78, 271 (1993).

[156] M. E. van Leeuwen: Deviation from corresponding-states behavior for polar fluids, Molec. Phys. 82, 383 (1994).

[157] M. J. Stevens, G. S. Grest: Phase coexistence of a Stockmayer fluid in an applied field, Phys. Rev. E 51, 5976 (1995).

[158] G. S. Rushbrooke, G. Stell, J. S. Høye: Theory of polar liquids: I. Dipolar hard spheres, Molec. Phys. 26, 1199 (1973).

[159] G. Stell, J. C. Rasaiah, H. Narang: Thermodynamic perturbation theory for simple polar fluids, Molec. Phys. 23, 393 (1972).

[160] K.-C. Ng, J. P. Valleau, G. M. Torrie: Liquid-vapor co-existence of dipolar hard spheres, Molec. Phys. 38, 781 (1979).

[161] J.-M. Caillol: Search of the gas-liquid transition of dipolar hard spheres J. Chem. Phys. 98, 9835 (1993).

[162] J. J. Weis, D. Levesque: Chain formation in low density dipolar hard spheres: A Monte Carlo study, Phys. Rev. Lett. 71, 2729 (1993).

[163] M. E. van Leeuwen, B. Smit: What makes a polar liquid a liquid?, Phys. Rev. Lett. 71, 3991 (1993).

[164] It should be noted however that the potential for $c \neq 0$ can still be written as Stockmayer potential with different $v_{0}$ and $D$.

[165] D. Levesque, J. J. Weis: Orientational and structural order in strongly interacting dipolar hard spheres, Phys. Rev. E 49, 5131 (1994).

[166] M. J. Stevens, G. S. Grest: Coexistence in dipolar fluids in a field, Phys. Rev. Lett. 72, 3686 (1994).

[167] P.-G. de Gennes, P. A. Pincus: Pair correlations in a ferromagnetic colloid, Phys. Kon- 
dens. Materie 11, 189 (1970).

[168] P. C. Jordan: Association phenomena in a ferromagnetic colloid, Molec. Phys. 25, 961 (1973).

[169] R. Rosman, J. J. M. Janssen, M. Th. Rekveldt: Interparticle correlations in $\mathrm{Fe}_{3} \mathrm{O}_{4}$ ferrofluids, studied by the small angle neutron scattering technique, J. Magn. Magn. Mater. 85, 97 (1990).

[170] K. Butter, P. H. H. Bomans, P. M. Frederik, G. J. Vroege, A. P. Philipse: Direct observation of dipolar chains in iron ferrofluids by cryogenic electron microscopy, Nature 2, 88 (2003).

[171] R. P. Sear: Low-density fluid phase of dipolar hard spheres, Phys. Rev. Lett. 76, 2310 (1996).

[172] R. van Roij: Theory of chain association versus liquid condensation, Phys. Rev. Lett. 76, 3348 (1996).

[173] M. A. Osipov, P. I. C. Teixeira, M. M. Telo da Gama: Structure of strongly dipolar fluids at low densities, Phys. Rev. E 54, 2597 (1996).

[174] J. M. Tavares, M. M. Telo da Gama, M. A. Osipov: Criticality of dipolar fluids: Liquid-vapor condensation versus phase separation in systems of living polymers, Phys. Rev. E 56, R6252 (1997).

[175] J. M. Tavares, J. J. Weis, M. M. Telo da Gama: Strongly dipolar fluids at low densities compared to living polymers, Phys. Rev. E 59, 4388 (1999).

[176] Y. Levin: What happened to the Gas-Liquid Transition in the system of dipolar hard spheres, Phys. Rev. Lett. 83, 1159 (1999).

[177] S. Klapp, F. Forstmann: Phase transitions in dipolar fluids: An integral equation study, J. Chem. Phys. 106, 9742 (1997).

[178] S. Klapp, F. Forstmann: Stability of ferroelectric fluid and solid phases in the Stockmayer model, Europhys. Lett. 38, 663 (1997).

[179] J. C. Shelley, G. N. Patey, D. Levesque, J. J. Weis: Liquid-vapor coexistence in fluids of dipolar hard dumbbells and spherocylinders, Phys. Rev. E 59, 3065 (1999).

[180] P. J. Camp, J. C. Shelley, G. N. Patey: Isotropic fluid phases of dipolar hard spheres, Phys. Rev. Lett. 84, 115 (2000).

[181] P. J. Camp, G. N. Patey: Structure and scattering in colloidal ferrofluids, Phys. Rev. E 62, 5403 (2000).

[182] A. F. Pshenichnikov, V. V. Mekhonoshin: Phase separation in dipolar systems: Numerical simulation, JETP Letters 72, 261 (2000). 
[183] A. F. Pshenichnikov, V. V. Mekhonoshin: Cluster structure and the first-order phase transition in dipolar systems, Eur. Phys. J. E 6, 399 (2001).

[184] K. I. Morozov, M. I. Shliomis: Magnetic fluid as an assembly of flexible chains, Lecture Notes in Physics 594, 162, edited by S. Odenbach, Springer Berlin Heidelberg (2002).

[185] S. Kantorovich, A. O. Ivanov: Formation of chain aggregates in magnetic fluids: an influence of polydispersity, J. Magn. Magn. Mater. 252, 244 (2002).

[186] P. G. Kusalik: Computer simulation study of a highly polar fluid under the influence of static electric fields, Molec. Phys. 81, 199 (1994).

[187] D. Boda, J. Winkelmann, J. Liszi, I. Szalai: Vapor-liquid equilibrium of Stockmayer fluids in applied field, Molec. Phys. 87, 601 (1996).

[188] I. Szalai, K.-Y. Chan, Y. K. Tang: Theoretical investigations of the vapor-liquid equilibrium and dielectric properties of dipolar Yukawa fluids in an external field, Molec. Phys. 101, 1819 (2003).

[189] S. Klapp, F. Forstmann: Phase behavior of dipolar hard spheres: Integral equations and density functional results, Phys. Rev. E 60, 3183 (1999).

[190] A. Yu. Zubarev, L. Yu. Iskakova: Theory of structural transformations in ferrofluids: Chain and "gas-liquid" phase transitions, Phys. Rev. E 65, 061406 (2002).

[191] L. Yu. Iskakova, A. Yu. Zubarev: Effect of interaction between chains on their size distribution: Strong magnetic field, Phys. Rev. E 66, 041405 (2002).

[192] H. Zhang, M. Widom: Spontaneous magnetic order in strongly coupled ferrofluids, J. Magn. Magn. Mater. 122, 119 (1993).

[193] P. Paffy-Muhoray, M. A. Lee, R. G. Petschek: Ferroelectric nematic liquid crystals: Realizability and molecular constraints, Phys. Rev. Lett 60, 2303 (1988).

[194] M. Baus, J.-L. Colot: Ferroelectric nematic liquid-crystals phases of dipolar hard ellipsoids, Phys. Rev. A 40, 5444 (1989).

[195] S. C. McGrother, G. Jackson: Islands of vapor-liquid coexistence in dipolar hard-core systems, Phys. Rev. Lett 76, 4183 (1996).

[196] D. C. Williamson, F. del Rio: The isotropic-nematic phase transition in a fluid of dipolar hard spherocylinders, J. Chem. Phys. 107, 9549 (1997).

[197] P. I. C. Teixeira, M. A. Osipov, M. M. Telo da Gama: Phase diagrams of aligned dipolar hard rods, Phys. Rev. E 57, 1752 (1998). 
[198] D. Wei, G. N. Patey: Orientational order in simple dipolar liquids: Computer simulation of a ferroelectric nematic phase, Phys. Rev. Lett 68, 2043 (1992).

[199] D. Wei, G. N. Patey: Ferroelectric liquid-crystal and solid phases formed by strongly interacting dipolar soft spheres, Phys. Rev. A 46, 7783 (1992).

[200] J. J. Weis, D. Levesque, G. J. Zarragoicoechea: Orientational order in simple dipolar liquidcrystal models, Phys. Rev. Lett. 69, 913 (1992).

[201] D. Wei, G. N. Patey, A. Perera: Orientational order in simple dipolar fluids: Densityfunctional theory and absolute-stability conditions, Phys. Rev. E 47, 506 (1993).

[202] B. Groh, S. Dietrich: Long-ranged orientational order in dipolar fluids, Phys. Rev. Lett. 72, 2422 (1994).

[203] M. Widom, H. Zhang: Comment on "Long-ranged orientational order in dipolar fluids", Phys. Rev. Lett. 74, 2616 (1995).

[204] B. Groh, S. Dietrich: Ferroelectric phases in Stockmayer fluids, Phys. Rev. E 50, 3814 (1994).

[205] H. Zhang, M. Widom: Global phase diagrams for dipolar fluids, Phys. Rev. E 49, R3591 (1994).

[206] B. Groh, S. Dietrich: Orientational order in dipolar fluids consisting of nonspherical hard particles, Phys. Rev. E 55, 2892 (1997).

[207] M. A. Osipov, P. I. C. Teixeira, M. M. Telo da Gama: Density-functional approach to the theory of dipolar fluids, J. Phys. A 30, 1953 (1997).

[208] A. O. Ivanov: Spontaneous ferromagnetic ordering in magnetic fluids, Phys. Rev. E 68, 011503 (2003).

[209] K. I. Morozov: Long-range order of dipolar fluids, J. Chem. Phys. 119, 13024-13032 (2003).

[210] B. J. Costa Cabral: Structure of polydisperse dipolar hard-sphere fluids J. Chem. Phys. 112, 4351 (2000).

[211] J. M. Tavares, J. M. Teixeira, M. M. Telo da Gama: The effect of dipolar forces on the structure and thermodynamics of classical fluids, J. Phys.:Condens. Matter 12, R411 (2000). 


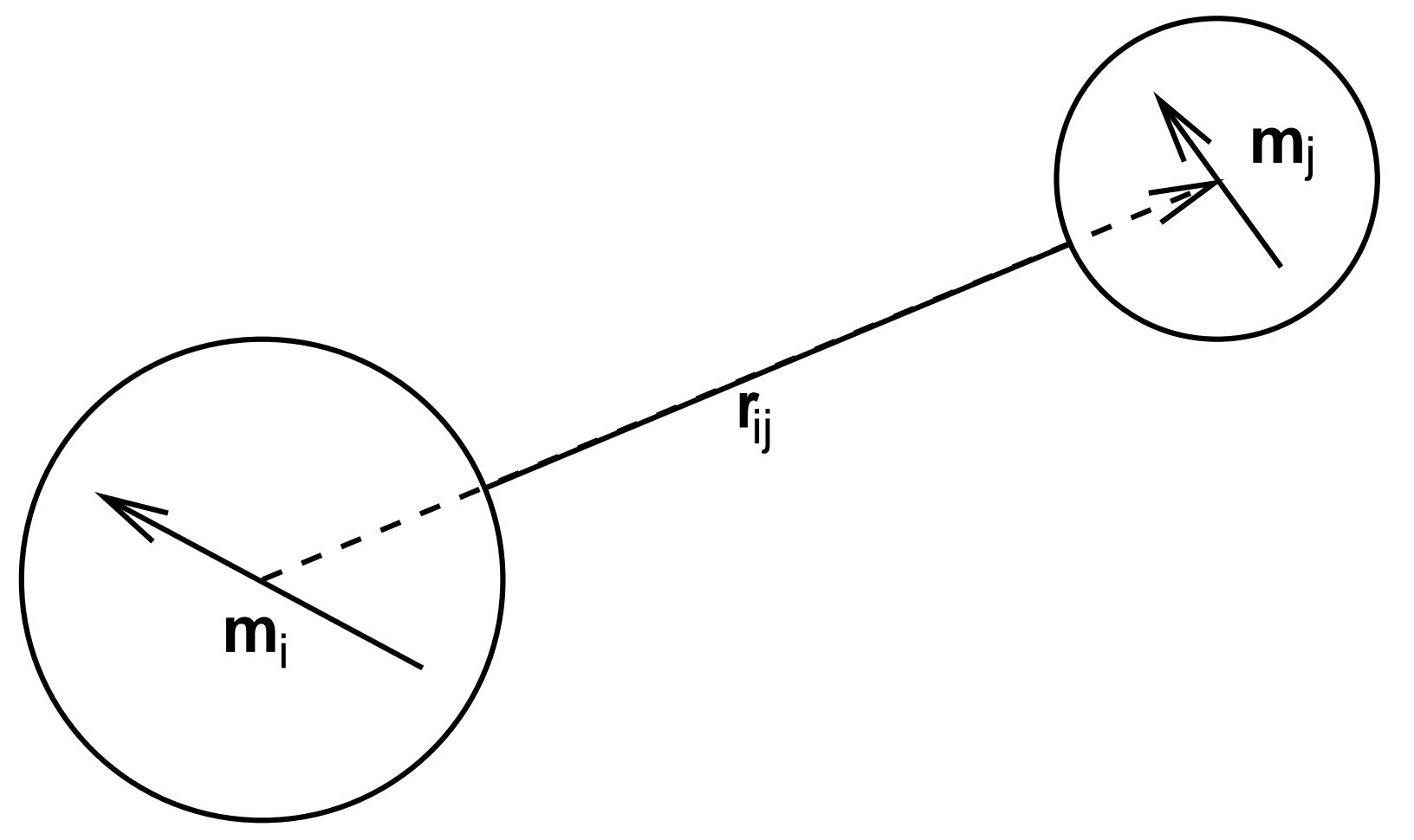

FIG. 1: Spheres $i$ and $j$ carrying dipole moments $\mathbf{m}_{i}$ and $\mathbf{m}_{j}$, respectively, that interact via the potential (2.2). 


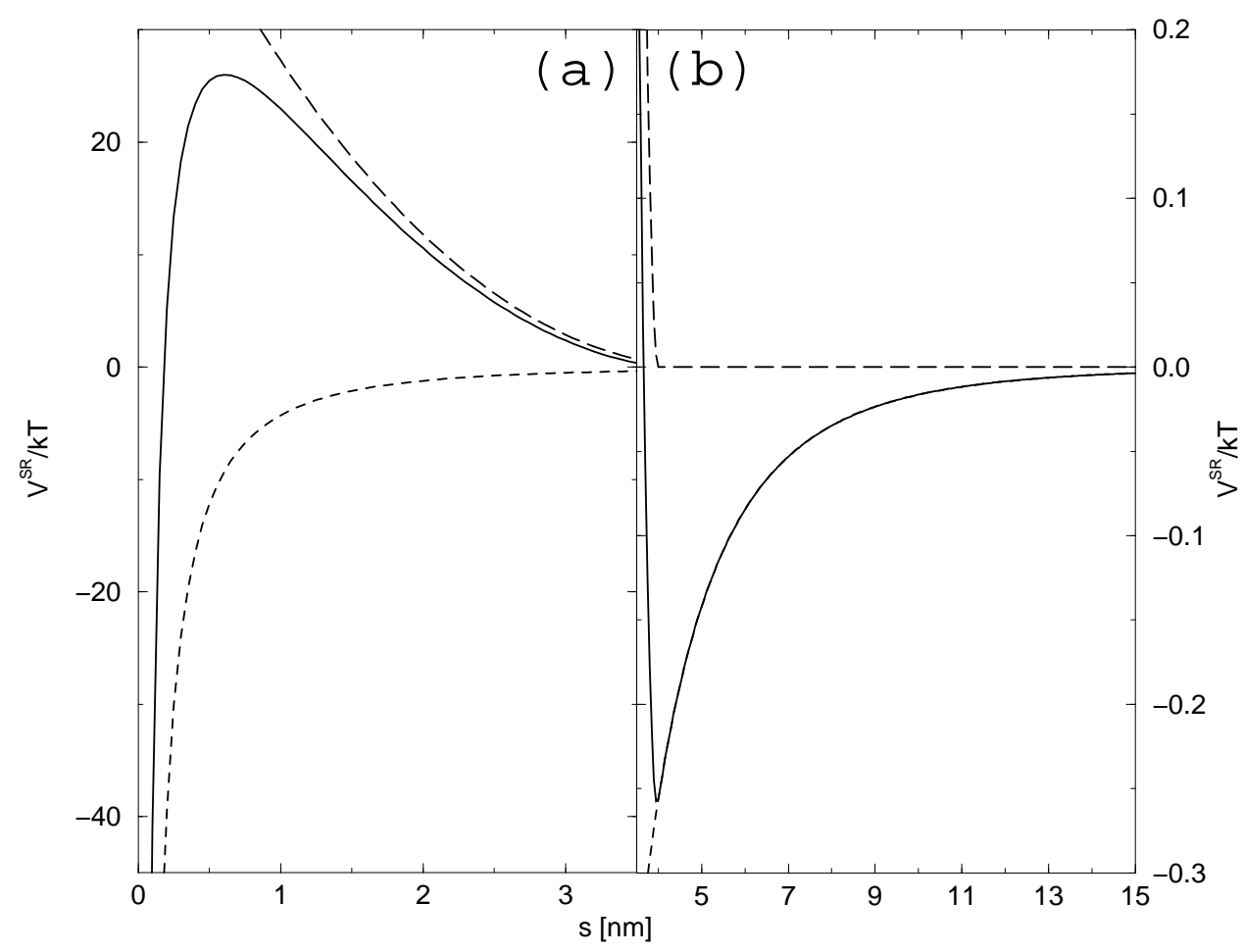

FIG. 2: Non-magnetic interaction potential for sterically stabilized ferrofluid particles as function of the surface-to-surface distance $s$. Short dashed line: van der Waals attraction, long dashed line: steric repulsion, solid line: combined potential. Note that the scales of both separation and energy are different in (a) and (b). 


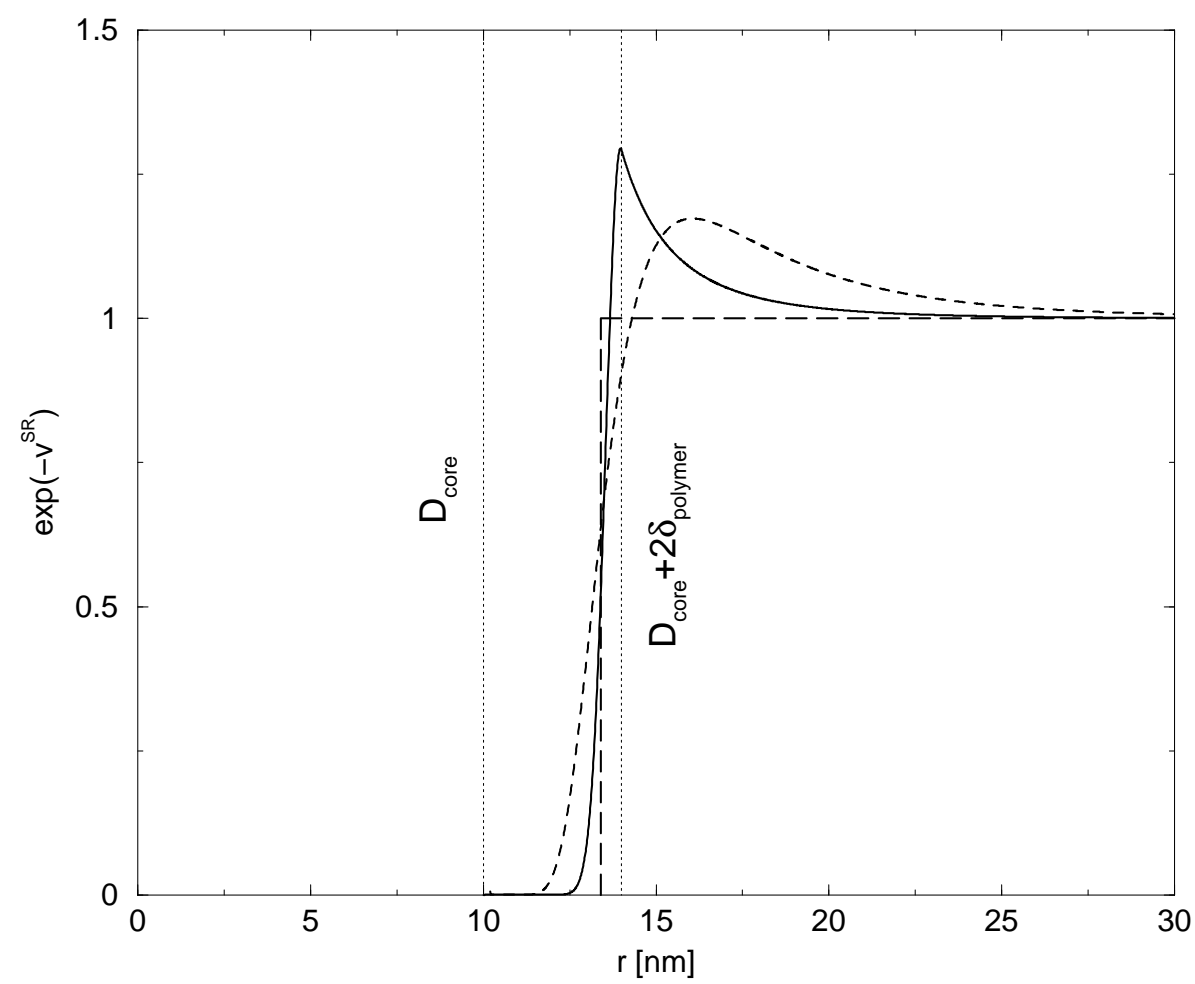

FIG. 3: The quantity $\exp \left(-v^{S R}\right)$ as function of particle distance for the potential of Fig. 2 (solid line). The contribution from the divergent part of the potential at very small surface-to-surface distances is suppressed in this plot. The short dashed and long dashed lines show $\exp \left(-v^{S R}(r)\right)$ for best fitting hard sphere and van der Waals potentials, respectively. 


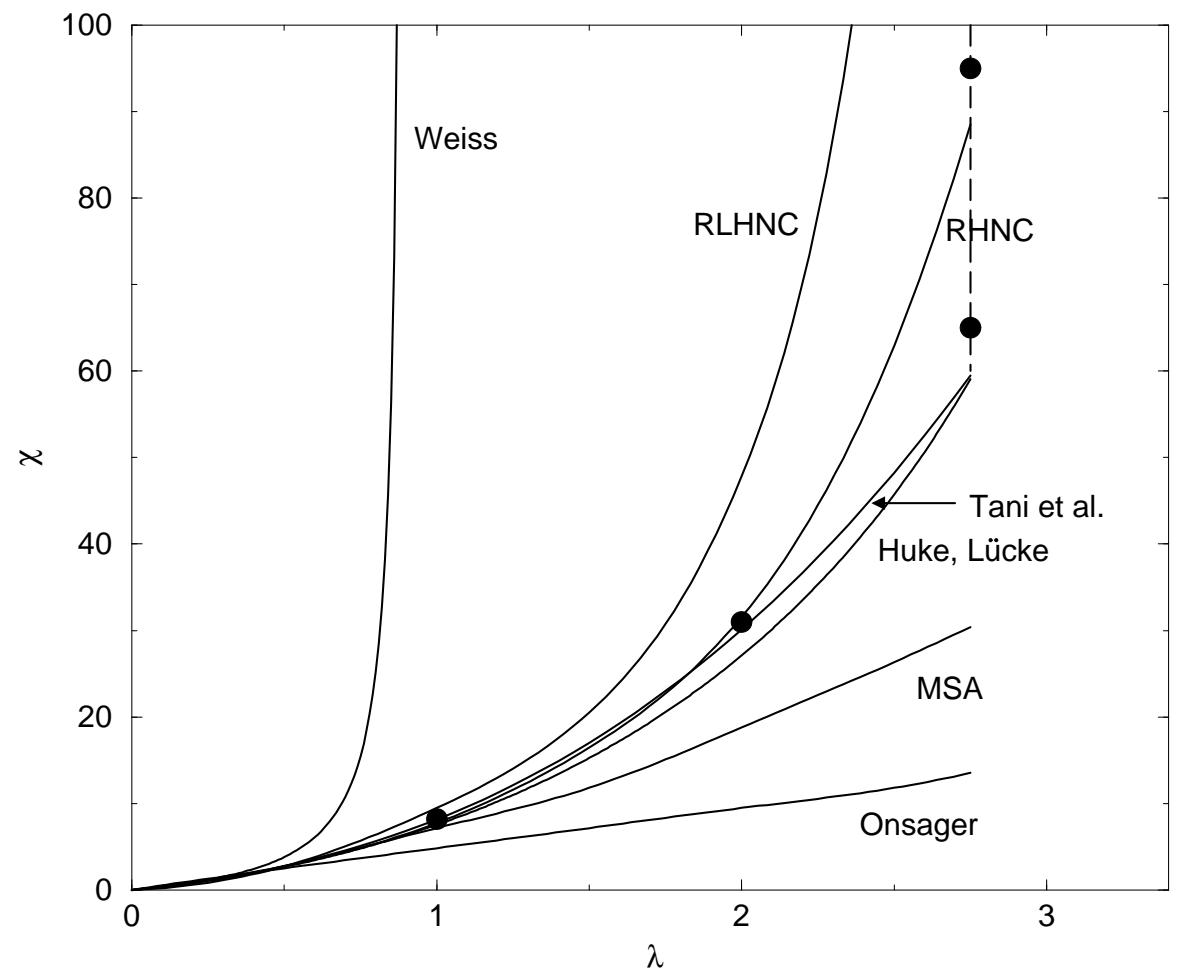

FIG. 4: Comparison of theoretical results for the initial susceptibility of dipolar hard spheres for $\phi=0.8 \pi / 6$ : Weiss [14] and Onsager [15] models, MSA by Wertheim [29], RLHNC results by Patey 34], RHNC results by Fries and Patey 39], cluster expansion result by Huke and Lücke 64], and the theory by Tani et al. 26]. Full circles denote Monte-Carlo data taken from a similar figure in [28]. 